\title{
Reduced Repetition Suppression in Aging is Driven by Tau-Related Hyperactivity in Medial Temporal Lobe
}

\author{
${ }^{\circledR}$ Jenna N. Adams, ${ }^{1}$ Anne Maass, ${ }^{1,2}{ }^{\circledR}$ David Berron, ${ }^{2,3}$ Theresa M. Harrison, ${ }^{1}{ }^{\circledR}$ Suzanne L. Baker, ${ }^{4}$ \\ Wesley P. Thomas, ${ }^{4}$ Morgan Stanfill, ${ }^{1}$ and ${ }^{\circledR}$ William J. Jagust ${ }^{1,4}$ \\ ${ }^{1}$ Helen Wills Neuroscience Institute, University of California, Berkeley, Berkeley, California 94720, ${ }^{2}$ German Center for Neurodegenerative Diseases \\ (DZNE), Magdeburg 39120, Germany, ${ }^{3}$ Clinical Memory Research Unit, Lund University, Lund 223 62, Sweden, and ${ }^{4}$ Lawrence Berkeley National \\ Laboratory, Berkeley, California 94720
}

Tau deposition begins in the medial temporal lobe (MTL) in aging and Alzheimer's disease (AD), and MTL neural dysfunction is commonly observed in these groups. However, the association between tau and MTL neural activity has not been fully characterized. We investigated the effects of tau on repetition suppression, the reduction of activity for repeated stimulus presentations compared to novel stimuli. We used task-based functional MRI (fMRI) to assess MTL subregional activity in 21 young adults (YA) and 45 cognitively normal human older adults (OA; total sample: 37 females, 29 males). AD pathology was measured with position emission tomography (PET), using ${ }^{18} \mathrm{~F}$-Flortaucipir for tau and ${ }^{11} \mathrm{C}$-Pittsburgh compound B (PiB) for amyloid- $\boldsymbol{\beta}(\mathrm{A} \beta)$. The MTL was segmented into six subregions using high-resolution structural images. We compared the effects of low tau pathology, restricted to entorhinal cortex and hippocampus (Tau- OA), to high tau pathology, also occurring in temporal and limbic regions (Tau + OA). Low levels of tau (Tau- OA vs YA) were associated with reduced repetition suppression activity specifically in anterolateral entorhinal cortex (alEC) and hippocampus, the first regions to accumulate tau. High tau pathology (Tau + vs Tau- OA) was associated with widespread reductions in repetition suppression across MTL. Further analyses indicated that reduced repetition suppression was driven by hyperactivity to repeated stimuli, rather than decreased activity to novel stimuli. Increased activation was associated with entorhinal tau, but not A $\beta$. These findings reveal a link between tau deposition and neural dysfunction in MTL, in which tau-related hyperactivity prevents deactivation to repeated stimuli, leading to reduced repetition suppression.

Key words: aging; Alzheimer's disease; fMRI; PET; repetition suppression; tau

Significance Statement

Abnormal neural activity occurs in the medial temporal lobe (MTL) in aging and Alzheimer's disease (AD). Because tau pathology first deposits in the MTL in aging, this altered activity may be due to local tau pathology, and distinct MTL subregions may be differentially vulnerable. We demonstrate that in older adults (OAs) with low tau pathology, there are focal alterations in activity in MTL subregions that first develop tau pathology, while OAs with high tau pathology have aberrant activity throughout MTL. Tau was associated with hyperactivity to repeated stimulus presentations, leading to reduced repetition suppression, the discrimination between novel and repeated stimuli. Our data suggest that tau deposition is related to abnormal activity in MTL before the onset of cognitive decline.

Received Sep. 24, 2020; revised Mar. 2, 2021; accepted Mar. 5, 2021.

Author contributions: J.N.A. and W.J.J. designed research; J.N.A, A.M., D.B., T.M.H., S.L.B., W.P.T., M.S., and W.J.J. performed research; J.N.A., A.M., D.B., T.M.H., S.L.B., W.P.T., and M.S. analyzed data; J.N.A. and W.J.J. wrote the paper.

This work was supported by National Institutes of Health Grants F31-AG062090 (to J.N.A.), F32-AG057107 (to T.M.H.), and R01-AG034570 (to W.J.J.); the Tau Consortium (W.J.J.); and the Helmholtz Postdoc Grant PD306 (to A.M.). Avid Radiopharmaceuticals enabled the use of the [18] Flortaucipir tracer but did not provide direct funding and were not involved in data analysis or interpretation.

W.J.J. serves as a consultant to Biogen, Genentech, CuraSen, Bioclinica, and Novartis. All other authors declare no competing financial interests.

Correspondence should be addressed to Jenna N. Adams at jnadams@berkeley.edu.

https://doi.org/10.1523/JNEUROSCI.2504-20.2021

Copyright $\odot 2021$ the authors

\section{Introduction}

Alzheimer's disease $(\mathrm{AD})$ is characterized by pathological aggregates of amyloid- $\beta(\mathrm{A} \beta)$ and hyperphosphorylated forms of the tau protein (Braak and Braak, 1991). These pathologies accumulate in the brains of cognitively normal older adults (OAs) 10-25 years before symptom onset and are measurable with positron emission tomography (PET; Schöll et al., 2016). While A $\beta$ pathology arises multifocally throughout association cortex, tau pathology first deposits in the medial temporal lobe (MTL), specifically in the transentorhinal region, before spreading to temporal and limbic cortex (Braak and Braak, 1985, 1991). 
Because the MTL is particularly vulnerable to tau pathology, determining how tau impacts the function and structure of this region is critical to understanding the transition from normal aging to disease. Abnormal neural activity in MTL regions such as entorhinal cortex and hippocampus has been found in OAs before evidence of cognitive decline (Yassa et al., 2011; Huijbers et al., 2014; Berron et al., 2018; Reagh et al., 2018), and thus may be an early response to pathology. Hippocampal hyperactivity, also found in mild cognitive impairment (MCI) (Dickerson et al., 2005; Yassa et al., 2010), has been shown to be dysfunctional, as its reduction leads to improved cognitive performance (Bakker et al., 2012).

Initial studies investigating pathology and MTL activity observed $\mathrm{A} \beta$-related hyperactivity primarily in the hippocampus (Sperling et al., 2009; Mormino et al., 2012a; Huijbers et al., 2014, 2015). However, these studies did not account for tau deposition, since tau-PET is a relatively recent technology. Because $\mathrm{A} \beta$ plaques do not accumulate in the MTL until late stages of $\mathrm{AD}$, tau may be more closely associated with abnormal MTL activity. Recent studies using PET or CSF measures of tau have supported stronger relationships between activation and tau than $\mathrm{A} \beta$ in hippocampus and cortical regions (Marks et al., 2017; Berron et al., 2019; Huijbers et al., 2019; Maass et al., 2019). However, a holistic characterization of how tau and/or A $\beta$ may differentially affect the activity of distinct subregions within MTL is needed to fully understand these relationships.

In the current study, we conduct a detailed analysis to determine whether tau and $\mathrm{A} \beta$ pathology, measured with PET, are associated with abnormal MTL activity in cognitively normal OAs. We expand on previous reports focusing on hippocampus (Marks et al., 2017; Huijbers et al., 2019) by measuring activity within six MTL subregions using high-resolution neuroimaging. We investigate for the first time how activity within regions that are highly vulnerable to $\mathrm{AD}$, such as entorhinal and perirhinal subregions, is related tau progression and entorhinal tau pathology using PET. We use functional MRI (fMRI) to assess MTL activity during repetition suppression, a phenomenon in which repeated stimuli show a dampened neural response as compared to novel stimuli, presumably as the stimulus is remembered and does not require new encoding (Grill-Spector et al., 2006). Repetition suppression is reduced in AD (Golby et al., 2005; Pihlajamäki et al., 2011), yet has been previously unexamined in relation to tau pathology.

We hypothesized that tau would be related to reduced repetition suppression activity, and that this association would occur in specific MTL subregions at different stages of tau pathological spread. We compared the effects of low tau pathology, restricted to entorhinal cortex and hippocampus and nearly ubiquitous in aging (Braak and Braak, 1997), to high tau pathology, which has spread throughout MTL, lateral temporal, and limbic regions. We hypothesized that the low tau group would have focal reductions in repetition suppression activity in the subregions that first accumulate tau: the anterolateral entorhinal cortex (alEC) and perirhinal area 35 (which span the transentorhinal region) and the hippocampus. In the high tau group, individuals at a more advanced stage of tau deposition, we expected to observe reduced repetition suppression activity across the MTL. Finally, due to the lack of $\mathrm{A} \beta$ plaques in MTL in normal aging, and in line with emerging findings, we hypothesized that $\mathrm{A} \beta$ would not be associated with MTL activation during repetition suppression.

\section{Materials and Methods}

\section{Participants}

Cognitively normal OAs and young adults (YAs) of either sex were recruited as part of the Berkeley Aging Cohort Study (BACS). Both OA and YA received high-resolution 3T structural and functional MRI during a memory task involving object and scene stimuli. A previous study in our lab used this fMRI data to investigate object and scene processing in anterior-temporal and posterior-medial brain networks (Maass et al., 2019). This previous study collapsed across trial-level performance, focusing on differences in activity between object and scene stimuli, and thus did not investigate task-related memory processes. Here, we used a subsample of $50 \mathrm{OAs}$ and 22 YAs who additionally received high-resolution T2 images necessary for MTL segmentation. We investigated MTL subregional activity during repetition suppression, focusing on activity during novel and repeated trial types. Three OA subjects had poor performance on the fMRI task (see below, fMRI task), and two OA and one YA had excessive motion during fMRI (see below, fMRI preprocessing); these subjects were excluded, resulting in a final sample of $45 \mathrm{OA}$ and 21 YA subjects. OA subjects additionally received tau-PET with ${ }^{18} \mathrm{~F}$-Flortaucipir, amyloid-PET with ${ }^{11} \mathrm{C}$-Pittsburgh Compound $\mathrm{B}$ (PiB), and neuropsychological assessment.

Inclusion criteria for the OA subjects included age of 60 or greater, cognitively normal status (Mini-Mental State Exam $\geq 25$ and neuropsychological scores within 1.5 SDs of age, education, and sex adjusted norms), no serious neurologic, psychiatric, or medial illness, no major contraindications found on MRI or PET, and independent living in the community. Inclusion criteria for YA subjects were no major health problems, no current or recent history of psychiatric illness, no history of neurologic disorders or traumatic brain injury, no substance abuse problems, no depression or antipsychotic medications, and fluency in English. The Institutional Review Boards of the University of California, Berkeley and the Lawrence National Laboratory approved this study. All participants provided written informed consent.

\section{Functional and structural MRI}

3T MRI acquisition

Structural and functional MRI was performed at the Henry H. Wheeler Jr. Brain Imaging Center with a 3T TIM/Trio scanner (Siemens Medical system, software version B17A) and a 32-channel head coil. High-resolution whole brain structural images were acquired using a T1-weighted volumetric magnetization prepared rapid gradient echo image (MPRAGE; voxel size $=1 \mathrm{~mm}$ isotropic, $\mathrm{TR}=2300 \mathrm{~ms}, \mathrm{TE}=2.98 \mathrm{~ms}$, matrix $=256 \times 240 \times$ $160, \mathrm{FOV}=256 \times 240 \times 160 \mathrm{~mm}^{3}$, sagittal plane, 160 slices, 5 -min acquisition time).

fMRI was then performed while subjects performed a mnemonic discrimination task (see Berron et al., 2018; details below). High-resolution whole-brain functional data were acquired using $\mathrm{T}_{2}{ }^{*}$-weighted gradientecho echoplanar images (GE-EPI; voxel size $=1.54 \mathrm{~mm}$ isotropic, multiband acceleration factor $4, \mathrm{TR}=2400 \mathrm{~ms}, \mathrm{TE}=37 \mathrm{~ms}$, flip angle $=45$, matrix $=138 \times 138, F o V=212 \times 212 \mathrm{~mm}^{2}$, interleaved acquisition, 88 slices, PA phase encoding, two 13 min runs). Two gradient echo images with different echo times were additionally collected to create a phase map for distortion correction (1.54-mm isotropic resolution, R-L encoding direction, $\mathrm{TR}=1000$, flip angle $=60, \mathrm{TE} 1=5.6 \mathrm{~ms}$, TE2 $=8.06 \mathrm{~ms}$ ).

To enable MTL subregion segmentation, high-resolution 3D T2weighted structural turbo spin echo images were acquired in oblique coronal orientation perpendicular to the long axis of the hippocampus ( voxel size $=0.5 \times 0.5 \mathrm{~mm}$ in-plane resolution, slice thickness $=1.5 \mathrm{~mm}$, $\mathrm{TR}=3500 \mathrm{~ms}, \mathrm{TE}=353 \mathrm{~ms}, 64$ slices, matrix $=384 \times 384$, GRAPPA factor 2 acceleration).

\subsection{T MRI acquisition and processing}

Structural MRIs were collected for standard PET processing at Lawrence Berkeley National Laboratory (LBNL). A whole brain high-resolution T1weighted volumetric magnetization prepared rapid gradient echo image (MPRAGE) was acquired on a 1.5T Siemens Magnetom Avanto scanner (Siemens, Inc; $1 \mathrm{~mm}$ isotropic voxels, $\mathrm{TR}=2110 \mathrm{~ms}, \mathrm{TE}=3.58 \mathrm{~ms}$, $\mathrm{FA}=15$ ). MRIs were used for PET coregistration, and segmented with FreeSurfer v.5.3.0 (http://surfer.nmr.mgh.harvard.edu/) to derive native space regions of interest (ROIs) for FTP and PiB quantification.

\section{fMRI task}

Subjects performed a mnemonic discrimination task during fMRI acquisition (Fig. 1A). Full details of the task have been described previously 

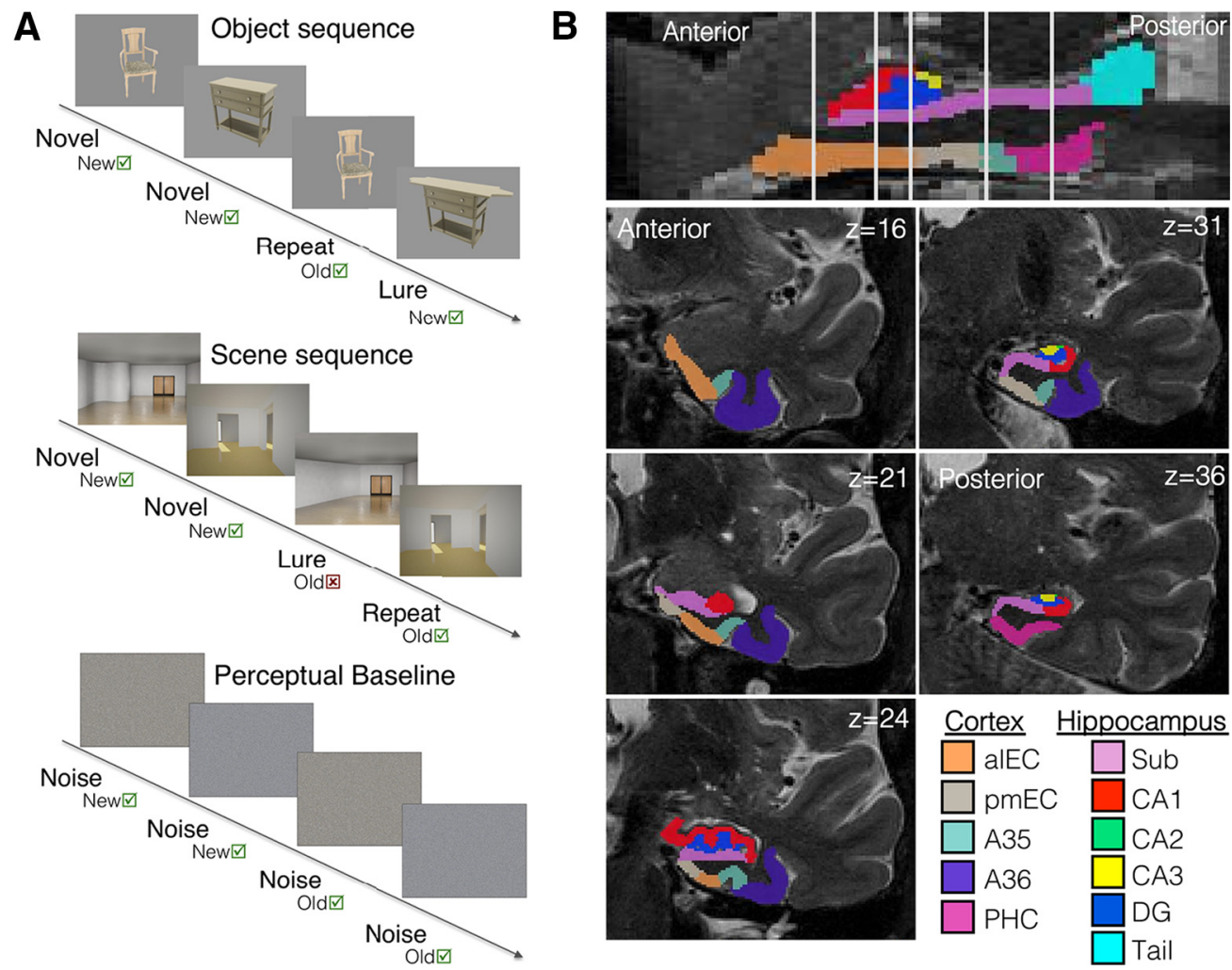

Figure 1. Visual representation of the fMRI task and MTL segmentation. $A, O A$ and YA subjects performed a mnemonic discrimination task during fMRI acquisition. The first two stimuli of each block (either object or scene sequences) are novel stimuli, while the second two stimuli are either repeated stimuli or highly similar lure stimuli. Subjects are instructed to respond old or new to each stimulus. A perceptual baseline of scrambled noise images was shown at the start and end of the task. Repetition suppression was measured by contrasting activity between all novel and all repeated stimuli. $\boldsymbol{B}$, High-resolution $(0.5 \times 0.5 \times 1.5 \mathrm{~mm})$ T2 images in native space were segmented with ASHS to derive MTL ROls for analysis. The full entorhinal cortex was manually subdivided into alEC and pmEC subregions using template ROls inverse warped to native space. Hippocampal subfields were merged into one hippocampal ROl before analyses. Cortical ROls: anterolateral entorhinal cortex (alEC), posteromedial EC (pmEC), area 35 of perirhinal cortex (A35), area 36 of perirhinal cortex (A36), parahippocampal cortex (PHC); hippocampal subfields: subiculum (Sub), CA1, CA2, CA3, dentate gyrus (DG), hippocampal tail (Tail).

(Berron et al., 2018; Maass et al., 2019). Briefly, subjects saw blocks of either four object or scene stimuli, where the first two stimuli each block were novel, and the second two stimuli were either repeated stimuli or highly similar lure stimuli. The subject was instructed to indicate "old" or "new" to each stimulus (repeated stimuli are considered old, novel/ lure stimuli are considered new). Before scanning, subjects completed a short training outside of the scanner. In the scanner, subjects completed two runs of the task, totaling 128 trials (64 first-repeat pairs, 64 first-lure pairs). Each run began and ended with a perceptual baseline condition, which consisted of scrambled noise images with similar luminosity and color to the test stimuli. Stimuli were presented in an event-related design using Neurobehavioral Systems (https://nbs.neurobs.com). Each object or scene image was shown for $3 \mathrm{~s}$ and separated by a white fixation star with jittered interstimulus intervals ranging from 0.6 to $4.2 \mathrm{~s}$. As a measure of task performance, we calculated the corrected hit rate (hit rate-false alarm rate) for all stimuli. Subjects were excluded if corrected hit rate was close to chance (corrected hit rate $<0.1$; Maass et al., 2019), leading to the exclusion of three OA subjects.

\section{fMRI preprocessing}

Preprocessing was conducted with Statistical Parametric Mapping (SPM, version 12, Wellcome Trust Center for Neuroimaging, London, United Kingdom). The first five EPIs of each run were discarded to ensure T1 equilibrium. Slice time correction was performed to correct for differences in acquisition, using the middle slice in time as a reference. Motion and distortion correction was then performed using the FieldMap toolbox v2.1 with the "realign and unwarp" SPM module. During this process, the T1 image was coregistered to the first EPI, and all EPIs were realigned to the first EPI image. EPIs were spatially smoothed with a Gaussian kernel of full-width at half-maximum (FWHM) $4 \mathrm{~mm}$ isotropic to increase signal-to-noise ratio while maintaining spatial specificity, consistent with previous studies (Berron et al., 2018, 2019). We next used the function "art.m" (CONN toolbox, originally developed within the ArtRepair toolbox) to detect outliers in average intensity and motion, using a global intensity $z$-score of 5 and a movement threshold of $0.9 \mathrm{~mm} / \mathrm{TR}$. Outlier volumes were included as spike regressors, or "censored," in the first-level design matrix (Lemieux et al., 2007; Power et al., 2015). A threshold of $20 \%$ outlier volumes across both runs was used to exclude subjects, resulting in the exclusion of one YA and two OA subjects. These motion thresholds are consistent with our previous work (Maass et al., 2019), and chosen as a compromise to identify problematic frames while still retaining as much data as possible with the propensity of OA subjects to motion during fMRI tasks.

\section{First level analysis}

First level analyses were conducted with SPM12. A general linear model (GLM) was performed including novel stimuli, repeated stimuli, and lure stimuli collapsed across response, but modeling object and scene stimuli separately. Scrambled noise images, as well as six motion regressors and outlier volumes, were also included as regressors. The two runs were concatenated before modeling. We applied an implicit mask of 0.8 of the global signal (SPM default threshold) to remove areas of low signal, which often include medial temporal regions. To calculate activation for repetition suppression, we contrasted all novel stimuli with all repeated stimuli, regardless of task performance. For subcomponent measures of novel and repeated stimuli, we contrasted each with the perceptual baseline (scrambled noise images). For follow-up analyses investigating the effects of stimulus type on repetition suppression, we repeated these contrasts but for object and scene stimuli separately. The mean $\beta$ value for each contrast image was calculated for each unilateral 
A

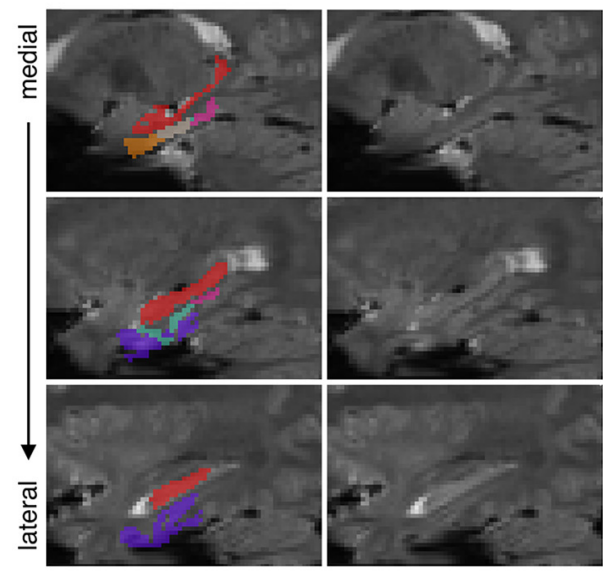

Example YA Subject

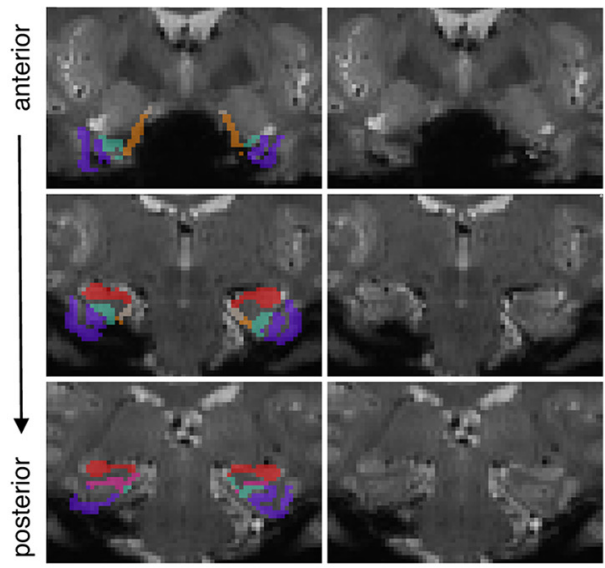

B

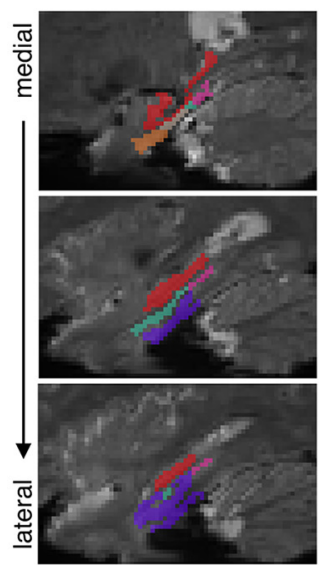

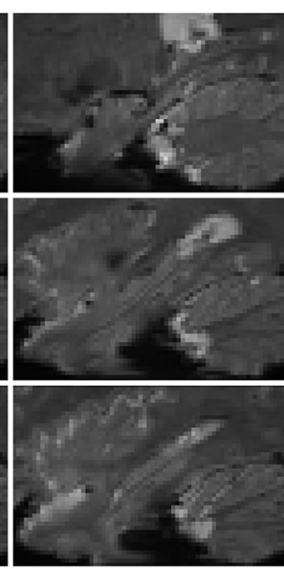

$\mathrm{PHC}$
Example OA Subject
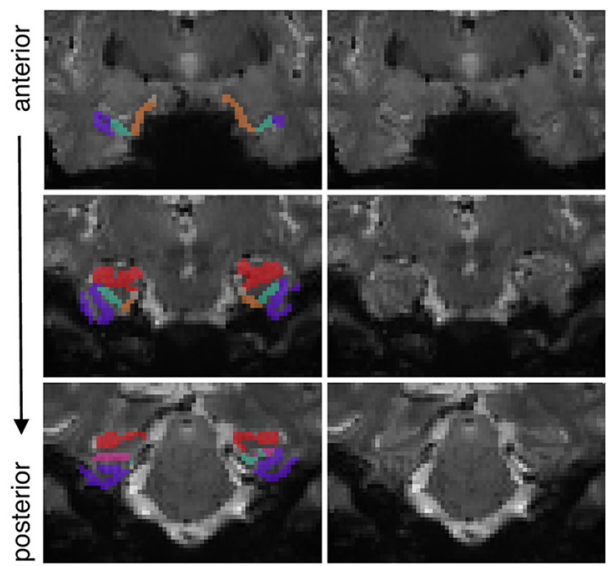

hipp

Figure 2. Example MTL segmentations and fMRI signal intensity. Representative images from a YA subject $(\boldsymbol{A})$ and OA subject $(\boldsymbol{B})$ are shown to visually demonstrate the quality of fMRI signal in each ROI. For each example subject, the MTL segmentation is overlaid on the mean functional image on the left column, and the mean functional image is shown by itself on the right column. Slices are shown sagittally from medial to lateral (left) and coronally from anterior to posterior (right). ROls: parahippocampal cortex (PHC), area 36 of perirhinal cortex (A36), area 35 of perirhinal cortex (A35), posteromedial EC (pmEC), anterolateral entorhinal cortex (alEC), hippocampus (hipp; all subfields merged). See Extended Data Figure 2-1 for corresponding proportions of voxels retained in each region in $\mathrm{YA}$ and $\mathrm{OA}$ groups after removing voxels of low signal.

MTL ROI (after removing additional regions of low signal, see below, MTL segmentation).

\section{MTL segmentation}

The MTL was automatically segmented into subregions with the software package automated segmentation of hippocampal subfields (ASHS; Yushkevich et al., 2015) using the high-resolution structural T2 image (Fig. 1B). Segmentation was based on a high-resolution atlas of the MTL trained on 38 individuals using 7T T2 structural images (Berron et al., 2017). This segmentation resulted in the following native space unilateral subregions: parahippocampal gyrus, area 36 of perirhinal cortex, area 35 of perirhinal cortex, entorhinal cortex, and hippocampal subfields (DG, CA1-CA3, subiculum, tail). All segmentations were manually inspected, and when necessary, edited for correctness.

Because of our interest in entorhinal subregions, the ASHS-derived entorhinal cortex was next segmented into anterolateral (alEC) and posteromedial (pmEC) subregions in a method similar to that of Berron et al. (2019). Because pure anatomic segmentation of the border between alEC and pmEC is challenging, we based our segmentation on template space alEC and pmEC ROIs that were functionally defined at $7 \mathrm{~T}$ in a previous study (for details, see Maass et al., 2015). First, we inversewarped these template ROIs into native space using ANTs (Avants et al.,
Table 1. Demographic characteristics of the sample

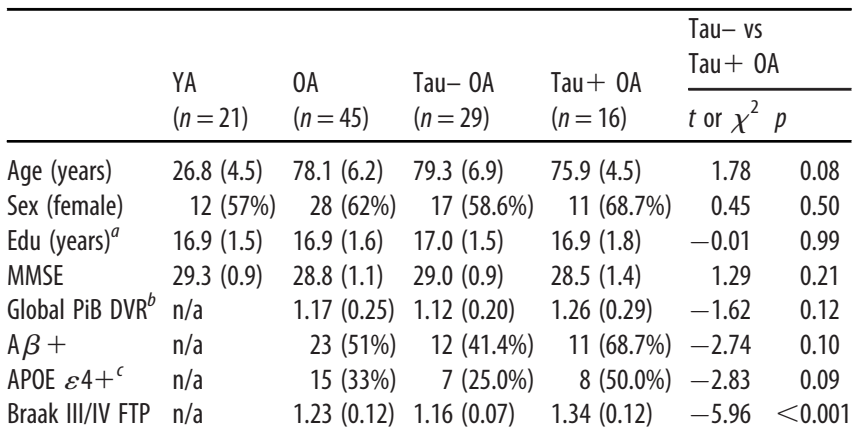

All values represent either $M(S D)$, or $N(\%)$. Demographics for the Tau- and Tau $+0 A$ groups were compared with independent $t$ tests or $\chi^{2}$ tests. Tau + status was determined by whether the mean Braak III/IV FTP value was $>1.26$. YA, young adults; $\mathrm{OA}$, older adults; MMSE, Mini-Mental State Examination. APOE, alolipoprotein $\mathrm{E}^{\mathrm{a}}$ four YA missing education; ${ }^{b}$ one $\mathrm{OA}$ missing PiB; ${ }^{C}$ one $\mathrm{OA}$ missing APOE.

2010). To do this, we created a study-specific template (separately for $\mathrm{YA}$ and OA groups), coregistered the original template space alEC/ pmEC ROIs to each study-specific template, and used ANTs to inverse warp the ROIs into native space for each subject. Next, we segmented 
A

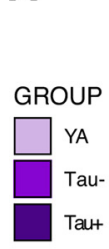

B

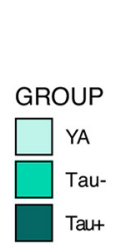

C

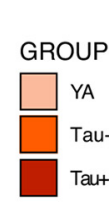

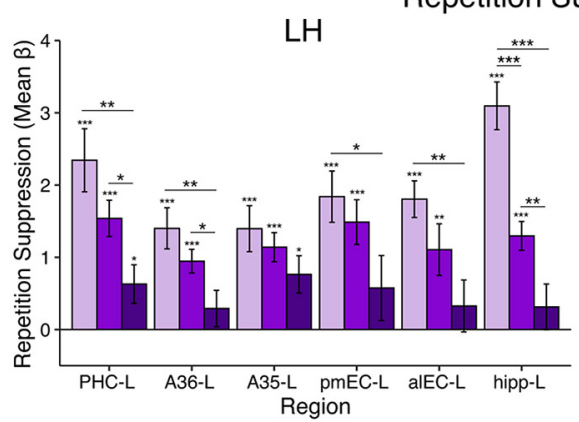

Repetition Suppression Activity

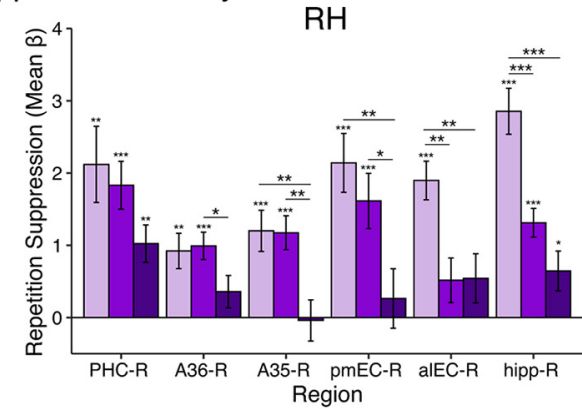

Novel Stimuli Activity
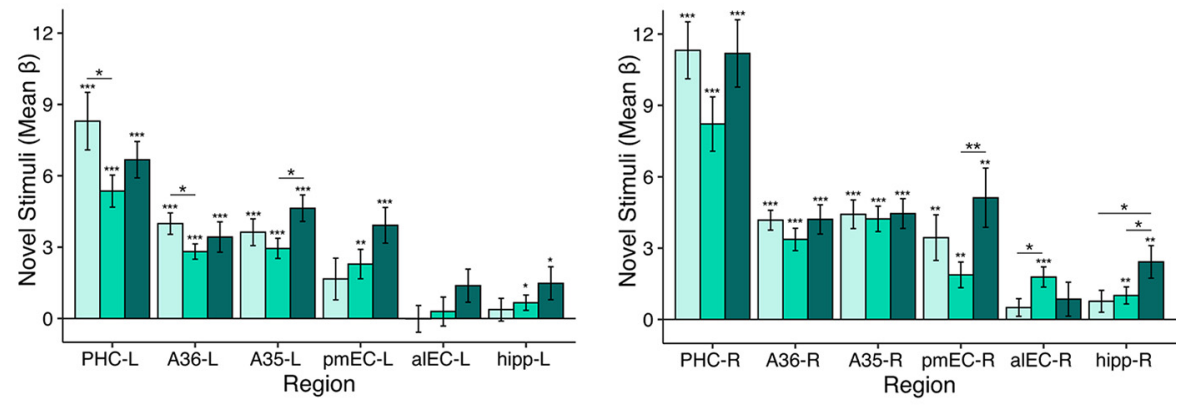

Repeated Stimuli Activity
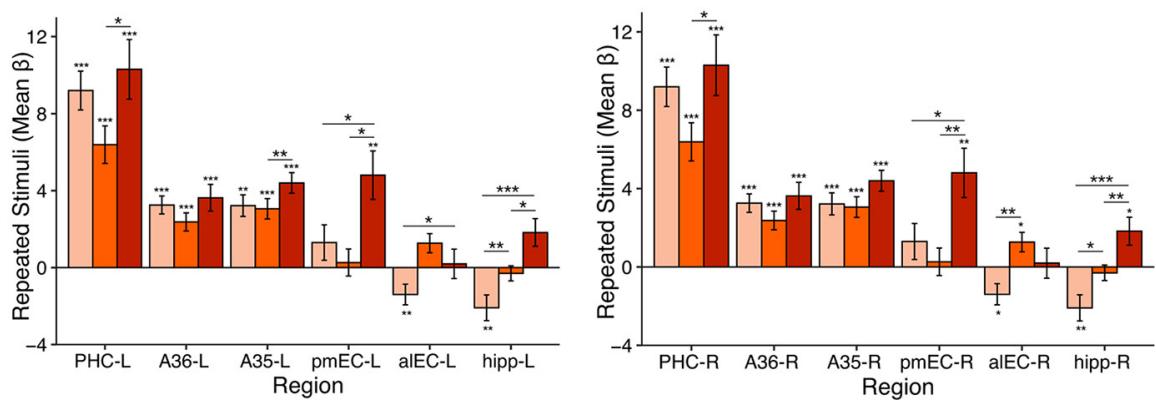

Figure 3. Tau-related differences in MTL activation for repetition suppression, novel stimuli, and repeated stimuli. $\boldsymbol{A}$, Repetition suppression was quantified by contrasting activity between novel and repeated stimuli, and compared across the YA, low tau (Tau- OA), and high tau (Tau + OA) groups with ANOVAs and follow-up pairwise comparisons. Tau- OA demonstrated a significant reduction in repetition suppression (vs YA) in bilateral hippocampus and alEC-R (independent samples $t$ test, $p s<0.05$ ). Tau $+0 \mathrm{~A}$ demonstrated additional reductions in repetition suppression across the MTL (vs YA and Tau- OA; $p s<0.05$ ). $B, C$, To determine whether activity changes to novel and/or repeated stimuli were driving reduced repetition suppression, we next investigated each subcomponent of the contrast separately. $\boldsymbol{B}$, Activity to novel stimuli was compared to the perceptual baseline condition. There were minimal group differences in activity to novel stimuli, including both increases and decreases in activity. C, Activity to repeated stimuli was compared to the perceptual baseline condition. Hyperactivity in the Tau- $0 \mathrm{~A}$ group (vs YA) was found in bilateral hippocampus and alEC-R, and hyperactivity in the Tau + OA group (vs Tau- OA and YA) was found throughout the MTL. This hyperactivity to repeated stimuli can be inferred to drive the reduction in repetition suppression seen in $\boldsymbol{A}$. Significance above each bar reflects one sample $t$ tests within each group, while lines across bars represent significant group differences with independent samples $t$ tests. Error bars represent SEM; $* * * p<0.001, * * p<0.01, * p<0.05$.

the ASHS-EC ROI into alEC and pmEC subregions using the division produced from the functional alEC/pmEC template ROIs. The original borders of the ASHS-EC ROI were retained, but at each coronal slice, we divided the EC ROI into alEC and pmEC based on the warped template ROIs. Voxels falling within the warped alEC/pmEC ROIs, but outside of the borders of the ASHS-EC ROI, were not retained in the final segmentation, however this occurred infrequently. Anatomical guidelines from Maass et al. (2015) describing the location of the functional alEC/pmEC template ROIs were also considered for accuracy and quality assurance of the inverse warping. In sum, this process resulted in each voxel of the original ASHS-EC ROI to be assigned to either alEC or pmEC.

We next postprocessed the MTL ROIs (including the new alEC/ pmEC subregions) for fMRI analyses. First, we merged the hippocampal subfields into one hippocampal ROI because of our resolution $(1.5 \mathrm{~mm}$ isotropic) not being ideal for a detailed examination of subfield activity, and to reduce the number of statistical tests, as we did not have subfieldspecific hypotheses about repetition suppression. Next, MTL ROIs were coregistered and resliced to functional image space using SPM. Finally, we additionally removed regions of low signal within each ROI because of concerns of signal drop out in MTL. This was performed by calculating the mean signal intensity across the entire bilateral MTL ROI for each subject, and removing any individual voxels that fell below 2 SDs of the mean (Libby et al., 2012; Maass et al., 2015). Figure 2 depicts an example YA and OA subject with their segmentation overlaid on their mean functional image to demonstrate signal quality within the MTL ROIs. Extended Data Figure 2-1 quantifies the average number of voxels retained after removing low signal voxels for each ROI in each age group. Additional implicit masking (as described above, First level analysis) was applied to these corrected ROIs during fMRI modeling to ensure that low signal voxels were not affecting our results.

\section{PET}

PET acquisition

All OA participants received PET at LBNL within an average of $66 \pm 108 \mathrm{~d}$ (minimum $=1 \mathrm{~d}$, maximum $=662 \mathrm{~d}$; Tau - OA $72 \pm 126 \mathrm{~d}$; $\mathrm{Tau}+\mathrm{OA} 54 \pm 68 \mathrm{~d}$ ) from the $3 \mathrm{~T}$ MRI session. Tau was measured with ${ }^{18} \mathrm{~F}$-Flortaucipir (FTP; previously known as ${ }^{18} \mathrm{~F}$-AV1451), which was synthesized at the Biomedical Isotope facility at LBNL (described in 
Schöll et al., 2016). Data were acquired on a BIOGRAPH PET/CT Truepoint six scanner (Siemens, Inc) from either 75-115 min postinjection or $0-100$ and $120-150$ min postinjection. All data were binned into $4 \times 5 \mathrm{~min}$ frames from 80 to $100 \mathrm{~min}$ postinjection. CT scans were acquired before the start of each emission acquisition. $\mathrm{A} \beta$ was measured using $\mathrm{PiB}$, synthesized at the Biomedical Isotope Facility at LBNL (Mathis et al., 2003). Data were acquired on either the same BIOGRAPH or an ECAT HR scanner (Siemens, Inc) across 35 dynamic frames for 90 min postinjection $(4 \times 15,8 \times 30,9 \times 60,2 \times 180,10 \times 300,2 \times 600$ s). A CT scan or PET transmission scan (ECAT HR) was acquired before emission acquisition. Images were reconstructed with an ordered subset expectation maximization algorithm, with attenuation correction, scatter correction, and smoothing with a Gaussian kernel of FWHM $4 \mathrm{~mm}$ isotropic.

\section{FTP processing}

FTP images were realigned, averaged, and coregistered to the participant's 1.5T structural MRI, consistent with standard PET processing in BACS. Standardized uptake value ratio (SUVR) images were calculated by averaging mean tracer uptake over the 80 - to 100 -min data, and normalized by an inferior cerebellar gray reference region (Baker et al., 2017). The mean SUVR of each native space FreeSurfer ROI was extracted and partial volume corrected using a modified Geometric Transfer Matrix approach (Rousset et al., 1998; Baker et al., 2017). We used partial volume corrected data to ensure that off-target FTP signal, specifically in choroid plexus, did not affect measures of FTP in entorhinal cortex and also to correct for the partial volume effect.

Two main FTP measures were used for analysis. First, to determine tau group status within our sample of $\mathrm{OA}$, we calculated a weighted mean SUVR within Braak III/IV regions, using an SUVR threshold of 1.26 (Maass et al., 2017). OA above this threshold (Tau+ OA) have high levels of tau within temporal and limbic regions (Braak III/IV), representing a more advanced stage of tau pathology. OA below this threshold (Tau- OA) have tau either restricted to entorhinal and hippocampal regions (Braak I/II) or no measurable tau pathology. Second, to explore specific associations between activation and entorhinal tau, we extracted the mean SUVR of the bilateral entorhinal cortex, derived from the FreeSurfer segmentation and partial volume corrected. We chose to use the FreeSurfer entorhinal ROI, rather than an ROI from our ASHS segmentation, because the our partial volume correction method has been validated using Freesurfer ROIs (Baker et al., 2017), and we were more confident that any signal bleed-in from off-target binding in choroid plexus would be attenuated.

\section{PiB processing}

$\mathrm{PiB}$ images were realigned. The average of the first $20 \mathrm{~min}$ of acquisition was used to calculate coregistration to the participant's $1.5 \mathrm{~T}$ structural MRI using SPM12. The coregistration parameters were then applied to the individual realigned frames. Distribution volume ratio (DVR) images were calculated with Logan graphical analysis over 35- to 90-min data, and normalized by a whole cerebellar gray reference region (Logan, 2000; Price et al., 2005). A measure of global PiB DVR was calculated from cortical FreeSurfer ROIs, and thresholded at 1.065 to determine amyloid positivity of the OA subjects (Mormino et al., 2012b; Villeneuve et al., 2015). One participant was missing PiB DVR data and was excluded from any $\mathrm{A} \beta$-related analyses.

\section{Cognitive data}

All OA performed a standard battery of neuropsychological assessments as part of normal BACS protocol. The testing session closest in time to the PET session was selected, occurring an average of $54 \mathrm{~d}$ (SD of $84 \mathrm{~d}$ ) from PET, and an average of $130 \mathrm{~d}$ (SD of $186 \mathrm{~d}$ ) from the $3 \mathrm{~T}$ fMRI session. Short and long delay free recall subtests from both the California Verbal Learning test (a measure of verbal memory) and the Visual Reproduction test (a measure of visual memory) were selected to compute a composite measure of episodic memory as previously described (Maass et al., 2018). Briefly, each subtest was $z$-scored on a larger sample of 155 cognitively normal OA subjects from BACS who received
Table 2. Repetition suppression activation and group differences with older adults divided into low (Tau-) and high (Tau + ) tau groups

\begin{tabular}{|c|c|c|c|c|c|c|c|c|c|}
\hline \multicolumn{10}{|c|}{ Activity within group } \\
\hline & \multicolumn{3}{|l|}{ YA } & \multicolumn{3}{|c|}{ Tau- OA } & \multicolumn{3}{|c|}{$\mathrm{Tau}+\mathrm{OA}$} \\
\hline & $t$ & df & $p$ & $t$ & $d f$ & $p$ & $t$ & $d f$ & $p$ \\
\hline PHC-L & 5.37 & 20 & $<0.001$ & 6.12 & 28 & $<0.001$ & 2.38 & 16 & 0.03 \\
\hline PHC-R & 5.53 & 20 & 0.001 & 5.53 & 28 & $<0.001$ & 3.95 & 16 & 0.001 \\
\hline A36-L & 4.9 & 20 & $<0.001$ & 5.78 & 28 & $<0.001$ & 1.16 & 16 & 0.27 \\
\hline$A 36-R$ & 5.23 & 20 & 0.001 & 5.23 & 28 & $<0.001$ & 1.60 & 16 & 0.13 \\
\hline A35-L & 4.40 & 20 & $<0.001$ & 5.65 & 28 & $<0.001$ & 2.97 & 16 & 0.01 \\
\hline A35-R & 5.01 & 20 & $<0.001$ & 5.01 & 28 & $<0.001$ & -0.14 & 16 & 0.89 \\
\hline pmEC-L & 5.19 & 20 & $<0.001$ & 4.81 & 28 & $<0.001$ & 1.28 & 16 & 0.22 \\
\hline pmEC-R & 4.22 & 20 & $<0.001$ & 4.22 & 28 & $<0.001$ & 0.64 & 16 & 0.53 \\
\hline alEC-L & 7.11 & 20 & $<0.001$ & 3.09 & 28 & 0.004 & 0.91 & 16 & 0.38 \\
\hline alEC-R & 1.68 & 20 & $<0.001$ & 1.68 & 28 & 0.10 & 1.60 & 16 & 0.13 \\
\hline hipp-L & 9.38 & 20 & $<0.001$ & 6.50 & 28 & $<0.001$ & 0.99 & 16 & 0.34 \\
\hline hipp-R & 6.62 & 20 & $<0.001$ & 6.62 & 28 & $<0.001$ & 2.35 & 16 & 0.03 \\
\hline \multicolumn{10}{|c|}{ Activity differences between groups } \\
\hline & \multicolumn{3}{|c|}{ YA vs Tau- OA } & \multicolumn{3}{|c|}{ YA vs Tau $+0 \mathrm{~A}$} & \multicolumn{3}{|c|}{$\underline{T a u}-\mathrm{vs} T a u+0 \mathrm{~A}$} \\
\hline & $t$ & df & $p$ & $t$ & $\mathrm{df}$ & $p$ & $t$ & $\mathrm{df}$ & $p$ \\
\hline PHC-L & 1.70 & 48 & 0.096 & 3.10 & 35 & 0.004 & 2.31 & 43 & 0.03 \\
\hline PHC-R & 0.49 & 48 & 0.63 & 1.87 & 28.62 & 0.071 & 1.66 & 43 & 0.06 \\
\hline A36-L & 1.39 & 32.84 & 0.18 & 2.82 & 35 & 0.008 & 2.27 & 43 & 0.03 \\
\hline A36-R & -0.23 & 48 & 0.82 & 1.65 & 35 & 0.109 & 2.08 & 43 & 0.04 \\
\hline A35-L & 0.71 & 48 & 0.48 & 1.48 & 35 & 0.149 & 1.13 & 43 & 0.26 \\
\hline A35-R & 0.07 & 48 & 0.94 & 3.02 & 35 & 0.005 & 3.20 & 43 & 0.003 \\
\hline pmEC-L & 0.75 & 48 & 0.48 & 2.24 & 35 & 0.031 & 1.71 & 43 & 0.09 \\
\hline pmEC-R & 0.93 & 48 & 0.36 & 3.18 & 35 & 0.003 & 2.25 & 43 & 0.03 \\
\hline alEC-L & 1.48 & 48 & 0.15 & 3.46 & 35 & 0.001 & 1.41 & 43 & 0.17 \\
\hline alEC-R & 3.23 & 48 & 0.002 & 3.18 & 35 & 0.003 & -0.05 & 43 & 0.96 \\
\hline hipp-L & 4.92 & 48 & $<0.001$ & 5.94 & 35 & $<0.001$ & 2.76 & 43 & 0.008 \\
\hline hipp-R & 4.32 & 48 & $<0.001$ & 5.06 & 35 & $<0.001$ & 1.99 & 43 & 0.05 \\
\hline
\end{tabular}

Activity within groups was assessed with one-sample $t$ tests. Activity differences between groups was assessed with independent samples $t$ tests. YA, young adults; Tau- OA, older adults with low tau; Tau + $O A$, older adults with high tau; $P H C$, parahippocampal cortex; $A 36$, area 36 of perirhinal cortex; $A 35$, area 35 of perirhinal cortex; pmEC, posteromedial entorhinal cortex; alEC, anterolateral entorhinal cortex; hipp, hippocampus.

neuropsychological testing, and the $z$-scores for each test were averaged to create one composite measure of episodic memory performance.

\section{Experimental design and statistical analyses}

Statistical analyses were performed using SPSS version 25 (IBM Corp, IBM SPSS Statistics, V25) and MATLAB version 2015a (The MathWorks, Inc). Demographic variables were compared between groups using independent samples $t$ tests for continuous variables, and $\chi^{2}$ tests for categorical variables. To characterize patterns of MTL activity within each group, we performed one-sample $t$ tests (two-tailed). To assess group differences in MTL activation, we performed repeated measures ANOVAs, with region (unilateral MTL ROIs) and hemisphere (right vs left) as withinsubjects factors, and group (YA vs Tau- vs Tau + OA) as a between-subjects factor. Degrees of freedom and corresponding p-values were corrected using Greenhouse-Geisser estimates in cases where sphericity was violated (Greenhouse and Geisser, 1959). Main effects and interactions were considered significant at $p<0.05$ (two-tailed). To further explore factors driving the main effects and interactions, follow-up pairwise comparisons were conducted with independent samples $t$ tests for each ROI, considered significant at $p<0.05$ (two-tailed). Hyperactivity for novel and repeated stimuli was compared with paired-samples $t$ tests (two-tailed).

For all correlational analyses, we computed skipped correlation coefficients with the "robust correlation toolbox" implemented in MATLAB (Wilcox, 2004; Pernet et al., 2013). This toolbox identifies bivariate outliers, which may bias traditional correlation estimates, by accounting for the overall structure of the data. Bivariate outliers are removed before performing skipped correlations, which reflect Pearson's $r$, and bootstrapped $95 \%$ confidence intervals (CIs) are produced. Correlations were 


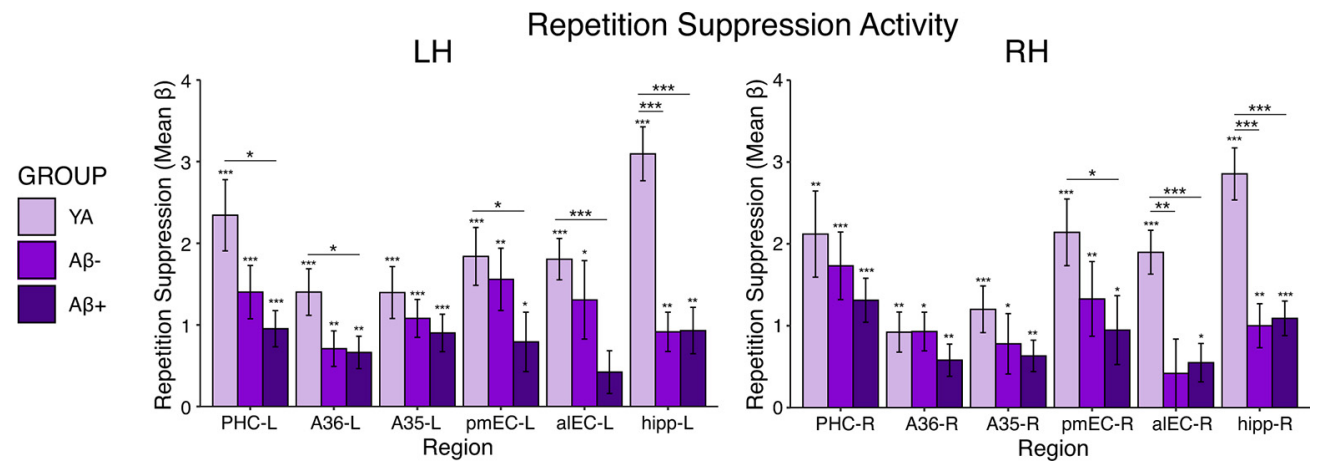

Figure 4. $A \beta$-related differences in MTL activation for repetition suppression. Repetition suppression activity was compared across the $Y A, A \beta-O A$, and $A \beta+O A$ groups with $A N O V A$ s and follow-up pairwise comparisons. While significant differences were found between the YA group and each $A \beta$ group, there were no additional significant difference between $A \beta$ - and $\mathrm{A} \beta+O \mathrm{~A}$, indicating no effect of $\mathrm{A} \beta$ positivity on repetition suppression activity ( $\mathrm{ps}>0.05$ ). Significance above each bar reflects one sample $t$ tests within each group, while lines across bars represent significant group differences with independent samples $t$ tests. Error bars represent SEM; $* * * p<0.001, * * p<0.01, * p<0.05$.

considered significant if the CI did not include zero. Because the toolbox does not support the inclusion of covariates, all covariates were regressed from the data before performing the skipped correlation.

\section{Results}

\section{Sample characteristics}

Table 1 reports demographic information for the YA and OA groups, as well as demographic comparisons between the low tau (Tau-) and high tau (Tau+) OA groups. Of our sample of 45 OA, 29 were classified as Tau- and 16 as Tau + based on FTP SUVR in Braak III/IV regions (Maass et al., 2017). By design, the Tau+ OA group had significantly higher mean Braak III/IV FTP SUVR than the Tau- OA group $(t=-5.96, p<0.001)$. No other demographic variables were significantly different between the tau groups, although there was a trend for older age in the TauOA group $(t=1.78, p=0.08)$, and more $\mathrm{A} \beta+\left(\chi^{2}=2.73\right.$, $p=0.10)$ and APOE $\varepsilon 4+$ subjects $\left(\chi^{2}=2.83, p=0.09\right)$ in the $\mathrm{Tau}+\mathrm{OA}$ group.

\section{Repetition suppression is reduced with tau pathology}

To obtain a measure of repetition suppression activity, we contrasted the activity of all novel stimuli (ignoring lure stimuli) against the activity of all repeated stimuli, extracting the mean $\beta$ weight of each unilateral MTL ROI. First, we aimed to establish which MTL ROIs demonstrated a repetition suppression effect, namely that repetition suppression activity (novel compared to repeated stimuli) was greater than zero, within each group using one-sample $t$ tests. In both the YA and Tau- OA groups, virtually all MTL ROIs showed repetition suppression $\beta$ values significantly greater than zero, confirming repetition suppression occurred in these ROIs (YA: $p s<0.001$; Tau- OA: ps $<0.01$; Fig. 3A; Table 2). In Tau $+\mathrm{OA}$, only the right hippocampus, left A35, and bilateral PHC demonstrated significant repetition suppression ( $p s<0.05$; Fig. $3 A$; Table 2 ); however, this group had a smaller sample size than the YA and Tau- OA groups and thus reduced power to observe significant within-group effects.

We next determined whether the groups differed in repetition suppression activity by conducting a repeated measures ANOVA, including region (MTL ROIs) and hemisphere (left vs right) as within-subjects factors and group (YA vs Tau-vs Tau+ OA) as a between-subjects factor. Our effects of interest were the main effect of group and group by region interactions, as these would suggest overall and regionally specific group differences, respectively. We found a significant main effect of group $\left(F_{(2)}=8.95\right.$, $p<0.001$ ), indicating that the amount of repetition suppression
Table 3. Repetition suppression activation and group differences with older adults divided by $A \beta$ positivity

\begin{tabular}{|c|c|c|c|c|c|c|c|c|c|}
\hline \multicolumn{10}{|c|}{ Activity within group } \\
\hline & \multicolumn{3}{|l|}{ YA } & \multicolumn{3}{|c|}{$A \beta-O A$} & \multicolumn{3}{|c|}{$\mathrm{A} \beta+0 \mathrm{~A}$} \\
\hline & $t$ & df & $p$ & $t$ & df & $p$ & $t$ & df & $p$ \\
\hline AC-L & 5.37 & 20 & $<0.001$ & 4.29 & 20 & $<0.001$ & 4.29 & 22 & $<0.001$ \\
\hline PHC-R & 4.03 & 20 & 0.001 & 4.20 & 20 & $<0.001$ & 4.87 & 22 & $<0.001$ \\
\hline A36-L & 4.92 & 20 & $<0.001$ & 3.26 & 20 & 0.004 & 3.34 & 22 & 0.003 \\
\hline A36-R & 3.77 & 20 & 0.001 & 3.93 & 20 & 0.01 & 2.93 & 22 & 0.008 \\
\hline A35-L & 4.40 & 20 & $<0.001$ & 4.66 & 20 & $<0.001$ & 3.92 & 22 & 0.001 \\
\hline A35-R & 4.21 & 20 & $<0.001$ & 2.11 & 20 & 0.047 & 3.29 & 22 & 0.003 \\
\hline mEC-L & 5.19 & 20 & $<0.001$ & 4.08 & 20 & 0.001 & 2.18 & 22 & 0.04 \\
\hline omEC-R & 5.26 & 20 & $<0.001$ & 2.92 & 20 & 0.009 & 2.25 & 22 & 0.04 \\
\hline alEC-L & 7.11 & 20 & $<0.001$ & 2.71 & 20 & 0.01 & 1.60 & 22 & 0.12 \\
\hline alEC-R & 7.09 & 20 & $<0.001$ & 1.00 & 20 & 0.33 & 2.34 & 22 & 0.03 \\
\hline hipp-L & 9.38 & 20 & $<0.001$ & 3.79 & 20 & 0.001 & 3.28 & 22 & 0.003 \\
\hline hipp-R & 8.97 & 20 & $<0.001$ & 3.72 & 20 & 0.001 & 5.16 & 22 & $<0.001$ \\
\hline \multicolumn{10}{|c|}{ Activity differences between groups } \\
\hline & \multicolumn{3}{|c|}{ YA vs $A \beta-O A$} & \multicolumn{3}{|c|}{ YA vs $A \beta+0 A$} & \multicolumn{3}{|c|}{$A \beta-v s A \beta+0 A$} \\
\hline & $t$ & $\mathrm{df}$ & $p$ & $t$ & $\mathrm{df}$ & $p$ & $t$ & df & $p$ \\
\hline AC-L & 1.73 & 40 & 0.09 & 2.19 & 42 & 0.006 & 1.81 & 42 & 0.26 \\
\hline IC-R & 0.58 & 40 & 0.56 & 1.37 & 29.9 & 0.18 & 0.87 & 42 & 0.39 \\
\hline $36-\mathrm{L}$ & 1.94 & 40 & 0.06 & 2.16 & 42 & 0.04 & 0.16 & 42 & 0.88 \\
\hline A36-R & -0.02 & 40 & 0.98 & 1.10 & 42 & 0.28 & 1.14 & 42 & 0.26 \\
\hline A35-L & 0.80 & 40 & 0.43 & 1.28 & 42 & 0.21 & 0.55 & 42 & 0.58 \\
\hline A35-R & 0.90 & 40 & 0.37 & 1.68 & 42 & 0.10 & 0.36 & 30.26 & 0.72 \\
\hline omEC-L & 0.54 & 40 & 0.59 & 2.06 & 42 & 0.046 & 1.45 & 42 & 0.15 \\
\hline ImEC-R & 1.33 & 40 & 0.19 & 2.03 & 42 & 0.049 & 0.61 & 42 & 0.54 \\
\hline AEC-L & 0.92 & 30.34 & 0.37 & 3.87 & 42 & $<0.001$ & 1.61 & 31.13 & 0.12 \\
\hline alEC-R & 2.98 & 40 & 0.005 & 3.80 & 42 & $<0.001$ & -0.28 & 42 & 0.78 \\
\hline hipp-L & 5.34 & 40 & $<0.001$ & 5.00 & 42 & $<0.001$ & -0.042 & 42 & 0.97 \\
\hline hipp-R & 4.45 & 40 & $<0.001$ & 4.69 & 42 & $<0.001$ & -0.27 & 42 & 0.79 \\
\hline
\end{tabular}

Activity within groups was assessed with one-sample $t$ tests. Activity differences between groups were assessed with independent samples $t$ tests. $A \beta-O A$, older adults who are $A \beta$ negative; $A \beta+O A$, older adults who are $A \beta$ positive; PHC, parahippocampal cortex; $A 36$, area 36 of perirhinal cortex; $A 35$, area 35 of perirhinal cortex; pmEC, posteromedial entorhinal cortex; alEC, anterolateral entorhinal cortex; hipp, hippocampus.

differed between groups regardless of MTL region. This corresponded to the stepwise pattern of repetition suppression found across the groups (Fig. 3A), with Tau- OA overall showing moderate reductions in repetition suppression, and Tau $+\mathrm{OA}$ showing more severe reductions in repetition suppression across the MTL relative to YA. We additionally found a significant main effect of region $\left(F_{(3.41)}=9.01, p<0.001\right)$, indicating that the amount of repetition suppression differed across regions regardless of group. 
A

B

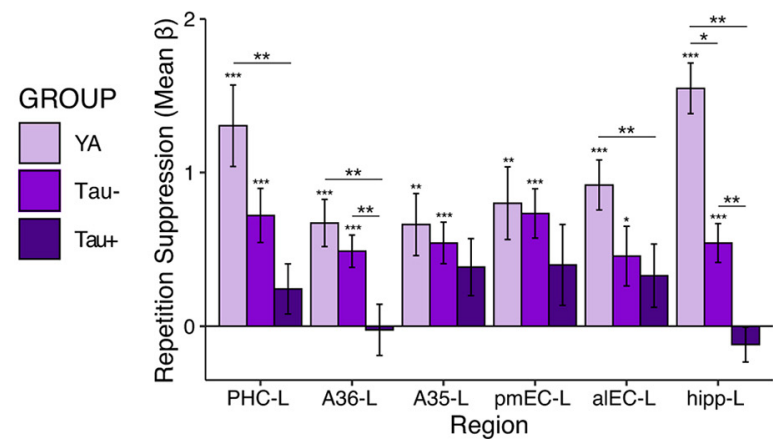

Objects
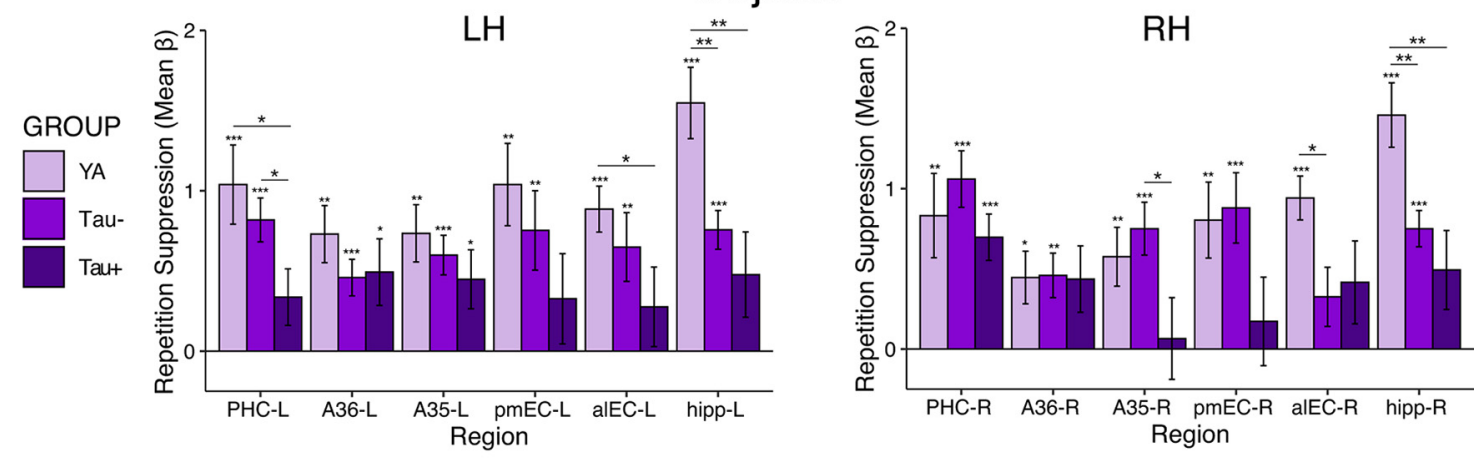

Scenes

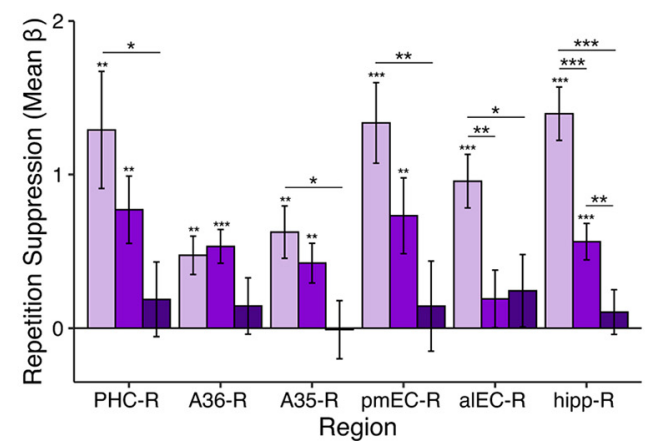

Figure 5. Tau-related differences in MTL activation for repetition suppression for object $(\boldsymbol{A})$ and scene $(\boldsymbol{B})$ stimuli modeled separately. Repetition suppression was defined as the difference in activity between the novel and repeated stimuli. Patterns of repetition suppression were generally similar for object $(\boldsymbol{A})$ and scene $(\boldsymbol{B})$ stimuli, both regarding patterns within each group and contrasts between groups. Significance above each bar reflects one sample $t$ tests within each age group, while lines across bars represent significant group differences with independent samples $t$ tests. Error bars represent SEM; $* * * p<0.001, * * p<0.01, * p<0.05$.

There was no significant main effect of hemisphere $\left(F_{(1)}=0.42\right.$, $p=0.52)$, or any interactions with hemisphere ( $p s>0.05)$. Finally, we found a significant region by group interaction $\left(F_{(6.827)}=3.55\right.$, $p=0.001$ ), indicating that the group difference in repetition suppression varied across MTL regions.

We further examined the region-specific group differences driving this interaction by conducting follow-up pairwise comparisons between the groups in each MTL region (Fig. $3 A$; Table 2 ), considered significant at $p<0.05$. To determine whether repetition suppression was reduced in OA with low levels of tau pathology, we first compared levels of repetition suppression activity in the Tau- OA group to the YA group. Tau- OA had significantly reduced repetition suppression compared to the YA group in the bilateral hippocampus and alEC-R $(p s<0.01)$. To determine whether a more advanced stage of tau pathology was associated with additional changes in repetition suppression, we next compared the Tau+ OA group to the Tau- OA group. $\mathrm{Tau}+\mathrm{OA}$ had significantly reduced repetition suppression compared with the Tau- OA group across many MTL regions, including the hipp-L, pmEC-R, A35-R, bilateral A36, and PHC-L $(p s<0.05)$. Finally, comparing the Tau + OA group to the YA group revealed widespread reductions in repetition suppression across virtually all MTL regions, including bilateral hippocampus, alEC, pmEC, A35-R, A36-L, and PHC-L ( $p s<0.05)$. Together, our findings suggest that low levels of tau deposition in aging are associated with focal decreases in repetition suppression in alEC and hippocampus, while high levels of tau pathology are associated with widespread decreases in repetition suppression throughout the MTL.

We repeated analyses comparing YA with $\mathrm{A} \beta-(n=21)$ and $\mathrm{A} \beta+(n=23)$ OA groups to determine whether the presence of
A $\beta$ also affected repetition suppression. We found a significant main effect of group $\left(F_{(2)}=6.22, p=0.003\right)$, main effect of region $\left(F_{(3.40)}=9.92, \quad p<0.001\right)$ and region by group interaction $\left(F_{(6.81)}=3.41, p=0.002\right)$, and no main effect of hemisphere $\left(F_{(1)}=0.59, p=0.44\right)$. However, follow-up pairwise comparisons indicated that while there were differences in repetition suppression activity between the YA group and each $\mathrm{A} \beta$ group, there were no significant group differences in repetition suppression activity between $\mathrm{A} \beta$ - and $\mathrm{A} \beta+\mathrm{OA}$ (Fig. 4; Table 3), suggesting that the presence of $\mathrm{A} \beta$, on its own, did not reduce repetition suppression.

Additionally, because MTL regions have been shown to demonstrate preference for processing object versus scene stimuli (Ranganath and Ritchey, 2012; Berron et al., 2018), we repeated the tau group analyses with repetition suppression activity calculated for the object and scene stimuli separately (Fig. 5). Overall, ANOVA models indicated significant main effects of group (objects: $F_{(2)}=3.02, p=0.06$; scenes: $F_{(2)}=8.18$, $p=0.001$ ) and region (object: $F_{(3.90)}=5.32, p<0.001$; scenes: $\left.F_{(3.52)}=4.40, p=0.003\right)$, though the main effect of group was trending for object stimuli. Critically, group by region interactions for object and scene stimuli separately were significant (object: $F_{(7.78)}=2.63, p=0.01$; scenes: $F_{(7.04)}=3.05$, $p=0.004)$. Follow-up pairwise comparisons revealed similar group differences for both the object and scene stimuli that were generally consistent with results from the combined stimuli. Low tau pathology (Tau- OA vs YA) was again associated with specific decreases in repetition suppression in bilateral hippocampus and alEC-R for both object and scene stimuli ( $p s<0.05)$. High tau pathology (Tau+ vs YA/Tau$\mathrm{OA}$ ) was associated with additional reductions in repetition 
Table 4. Novel stimuli activation and group differences with older adults divided into low (Tau-) and high (Tau + ) tau groups

\begin{tabular}{|c|c|c|c|c|c|c|c|c|c|}
\hline \multicolumn{10}{|c|}{ Activity within group } \\
\hline & \multicolumn{3}{|l|}{ YA } & \multicolumn{3}{|l|}{ Tau- } & \multicolumn{3}{|l|}{ Tau +} \\
\hline & $t$ & df & $p$ & $t$ & df & $p$ & $t$ & $\mathrm{df}$ & $p$ \\
\hline PHC-L & 6.85 & 20 & $<0.001$ & 7.97 & 28 & $<0.001$ & 8.73 & 15 & $<0.001$ \\
\hline PHC-R & 9.43 & 20 & $<0.001$ & 7.20 & 28 & $<0.001$ & 7.91 & 15 & $<0.001$ \\
\hline A36-L & 8.83 & 20 & $<0.001$ & 8.80 & 28 & $<0.001$ & 5.38 & 15 & $<0.001$ \\
\hline$A 36-R$ & 9.99 & 20 & $<0.001$ & 7.14 & 28 & $<0.001$ & 6.85 & 15 & $<0.001$ \\
\hline A35-L & 6.44 & 20 & $<0.001$ & 6.97 & 28 & $<0.001$ & 8.34 & 15 & $<0.001$ \\
\hline A35-R & 7.36 & 20 & $<0.001$ & 7.94 & 28 & $<0.001$ & 7.13 & 15 & $<0.001$ \\
\hline pmEC-L & 1.90 & 20 & 0.07 & 3.71 & 28 & 0.001 & 5.23 & 15 & $<0.001$ \\
\hline pmEC-R & 3.59 & 20 & 0.002 & 3.47 & 28 & 0.002 & 4.11 & 15 & 0.001 \\
\hline alEC-L & -0.03 & 20 & 0.98 & 0.48 & 28 & 0.63 & 1.98 & 15 & 0.07 \\
\hline alEC-R & 1.36 & 20 & 0.19 & 4.23 & 28 & $<0.001$ & 1.20 & 15 & 0.25 \\
\hline hipp-L & 0.78 & 20 & 0.45 & 2.07 & 28 & 0.048 & 2.13 & 15 & 0.05 \\
\hline$p-R$ & 1.67 & 20 & 0.111 & 2.83 & 28 & 0.009 & 3.54 & 15 & 0.003 \\
\hline \multicolumn{10}{|c|}{ Activity differences between groups } \\
\hline & \multicolumn{3}{|c|}{ YA vs Tau- OA } & \multicolumn{3}{|c|}{ YA vs Tau $+0 \mathrm{~A}$} & \multicolumn{3}{|c|}{$\underline{T a u}-$ vs Tau+OA } \\
\hline & $t$ & df & $p$ & $t$ & $\mathrm{df}$ & $p$ & $t$ & $\mathrm{df}$ & $p$ \\
\hline IC-L & 2.27 & 48 & & 1.05 & 35 & 0.30 & -1.23 & 43 & 22 \\
\hline PHC-R & 1.84 & 48 & 0.07 & 0.07 & 35 & 0.94 & -1.59 & 43 & 0.12 \\
\hline A36-L & 2.18 & 48 & 0.03 & 0.74 & 35 & 0.46 & -0.96 & 43 & 0.34 \\
\hline A36-R & 1.23 & 48 & 0.23 & -0.04 & 35 & 0.97 & -1.08 & 43 & 0.29 \\
\hline A35-L & 0.98 & 48 & 0.33 & -1.25 & 35 & 0.22 & -2.40 & 43 & 0.02 \\
\hline$A 35-R$ & 0.23 & 48 & 0.82 & -0.04 & 35 & 0.97 & -0.26 & 43 & 0.80 \\
\hline pmEC-L & -0.60 & 48 & 0.55 & -1.88 & 35 & 0.07 & -1.63 & 43 & 0.11 \\
\hline pmEC-R & 1.52 & 48 & 0.14 & -1.09 & 35 & 0.29 & -2.76 & 43 & 0.008 \\
\hline alEC-L & -0.36 & 48 & 0.72 & -1.58 & 35 & 0.12 & -1.12 & 43 & 0.27 \\
\hline alEC-R & -2.17 & 48 & 0.04 & -0.47 & 35 & 0.65 & 1.20 & 43 & 0.24 \\
\hline hipp-L & -0.53 & 48 & 0.60 & -1.36 & 35 & 0.18 & -1.07 & 21.535 & 0.30 \\
\hline hipp-R & -0.44 & 48 & 0.67 & -2.09 & 35 & 0.04 & -2.02 & 43 & 0.05 \\
\hline
\end{tabular}

Activity within groups was assessed with one-sample $t$ tests. Activity differences between groups was assessed with independent samples $t$ tests. YA, young adults; Tau $-0 \mathrm{~A}$, older adults with low tau; Tau+ OA, older adults with high tau; PHC, parahippocampal cortex; A36, area 36 of perirhinal cortex; $A 35$, area 35 of perirhinal cortex; pmEC, posteromedial entorhinal cortex; alEC, anterolateral entorhinal cortex; hipp, hippocampus.

suppression across MTL $(p s<0.05)$. Although separate object and scene analyses have reduced power because of fewer trials included in the estimation of each mean $\beta$ weight, these analyses generally replicated the overall pattern and conclusions found when collapsing across both object and scene stimuli, suggesting these effects are not specific to one type of stimuli within the MTL.

\section{Reduced repetition suppression is driven by hyperactivity to repeated stimuli}

Reduced repetition suppression in the OA groups may result from two different underlying mechanisms: (1) reduction in activity to novel stimuli, with no difference in repeated stimuli; or (2) hyperactivity to repeated stimuli, with no difference in novel stimuli. To further understand how tau pathology was related to changes in repetition suppression activity, we next sought to determine which pattern was driving our results by using the perceptual baseline condition in the task (Fig. 1A). For each MTL ROI, we extracted activity to novel stimuli compared to the perceptual baseline, and activity to repeated stimuli compared to the perceptual baseline. Because our original repetition suppression contrast did not include the perceptual baseline while calculating the contrast (a direct contrast between novel and repeated stimuli), a simple subtraction of these subcomponent $\beta$ weights (novel vs baseline - repeated
Table 5. Repeated stimuli activation and group differences with older adults divided into low (Tau-) and high (Tau + ) tau groups

\begin{tabular}{|c|c|c|c|c|c|c|c|c|c|}
\hline \multicolumn{10}{|c|}{ Activity within group } \\
\hline & \multicolumn{3}{|l|}{ YA } & \multicolumn{3}{|c|}{ Тau- OA } & \multicolumn{3}{|c|}{$\mathrm{Tau}+\mathrm{OA}$} \\
\hline & $t$ & df & $p$ & $t$ & df & $p$ & $t$ & $d f$ & $p$ \\
\hline HC-L & 5.45 & 20 & $<0.001$ & 6.41 & 28 & $<0.001$ & 7.07 & 15 & $<0.001$ \\
\hline PHC-R & 9.14 & 20 & $<0.001$ & 6.55 & 28 & $<0.001$ & 6.65 & 15 & $<0.001$ \\
\hline A36-L & 5.77 & 20 & $<0.001$ & 5.23 & 28 & $<0.001$ & 4.83 & 15 & $<0.001$ \\
\hline A36-R & 6.92 & 20 & $<0.001$ & 5.01 & 28 & $<0.001$ & 5.23 & 15 & $<0.001$ \\
\hline A35-L & 4.07 & 20 & 0.001 & 4.48 & 28 & $<0.001$ & 6.71 & 15 & $<0.001$ \\
\hline A35-R & 5.75 & 20 & $<0.001$ & 5.81 & 28 & $<0.001$ & 8.25 & 15 & $<0.001$ \\
\hline pmEC-L & -0.20 & 20 & 0.84 & 1.23 & 28 & 0.23 & 3.54 & 15 & 0.003 \\
\hline pmEC-R & 1.42 & 20 & 0.17 & 0.38 & 28 & 0.71 & 3.82 & 15 & 0.002 \\
\hline alEC-L & -2.98 & 20 & 0.007 & -1.01 & 28 & 0.322 & 0.93 & 15 & 0.37 \\
\hline alEC-R & -2.60 & 20 & 0.02 & 2.56 & 28 & 0.016 & 0.25 & 15 & 0.80 \\
\hline hipp-L & -4.06 & 20 & 0.001 & -1.68 & 28 & 0.10 & 1.59 & 15 & 0.13 \\
\hline$p-R$ & -3.15 & 20 & 0.005 & -0.76 & 28 & 0.45 & 2.55 & 15 & 0.02 \\
\hline \multicolumn{10}{|c|}{ Activity differences between groups } \\
\hline & \multicolumn{3}{|c|}{ YA vs Tau $-0 \mathrm{~A}$} & \multicolumn{3}{|c|}{ YA vs Tau $+0 \mathrm{~A}$} & \multicolumn{3}{|c|}{$\underline{\text { Tau }- \text { vs Tau }+0 \mathrm{~A}}$} \\
\hline & $t$ & $\mathrm{df}$ & $p$ & $t$ & $\mathrm{df}$ & $p$ & $t$ & $\mathrm{df}$ & $p$ \\
\hline HC-L & 1.84 & 48 & 0.07 & -0.10 & 35 & 0.92 & -2.22 & 43 & 0.03 \\
\hline PHC-R & 1.96 & 48 & 0.06 & -0.62 & 35 & 0.54 & -2.24 & 43 & 0.03 \\
\hline A36-L & 1.27 & 48 & 0.21 & -0.50 & 35 & 0.62 & -1.65 & 43 & 0.11 \\
\hline A36-R & 1.28 & 48 & 0.21 & -0.46 & 35 & 0.65 & -1.53 & 43 & 0.13 \\
\hline A35-L & 0.64 & 48 & 0.53 & -1.97 & 35 & 0.06 & -2.91 & 43 & 0.006 \\
\hline A35-R & 0.21 & 48 & 0.84 & -1.48 & 35 & 0.15 & -1.65 & 43 & 0.11 \\
\hline pmEC-L & -0.91 & 48 & 0.37 & -2.61 & 35 & 0.01 & -2.17 & 43 & 0.04 \\
\hline pmEC-R & 0.92 & 48 & 0.36 & -2.31 & 35 & 0.03 & -3.43 & 43 & 0.001 \\
\hline alEC-L & -0.94 & 48 & 0.36 & -2.58 & 35 & 0.01 & -1.27 & 43 & 0.21 \\
\hline alEC-R & -3.60 & 48 & 0.001 & -1.75 & 35 & 0.09 & 1.23 & 43 & 0.25 \\
\hline hipp-L & -2.90 & 48 & 0.006 & -3.91 & 35 & $<0.001$ & -2.41 & 43 & 0.02 \\
\hline hipp-R & -2.45 & 48 & 0.02 & -3.98 & 35 & $<0.001$ & -2.84 & 43 & 0.007 \\
\hline
\end{tabular}

Activity within groups was assessed with one-sample $t$ tests. Activity differences between groups was assessed with independent samples $t$ tests. YA, young adults; Tau- OA, older adults with low tau; Tau+ $O A$, older adults with high tau; $P H C$, parahippocampal cortex; $A 36$, area 36 of perirhinal cortex; $A 35$, area 35 of perirhinal cortex; pmEC, posteromedial entorhinal cortex; alEC, anterolateral entorhinal cortex; hipp, hippocampus.

vs baseline) may not result in an identical "repetition suppression" $\beta$ weight.

We first compared activity to novel stimuli across the groups with repeated measures ANOVAs (Fig. $3 B$ ). We did not observe a significant main effect of group $\left(F_{(2)}=2.25, p=0.12\right)$, indicating that overall, the groups did not differ in activation to novel stimuli. However, we did find a significant main effect of region $\left(F_{(2.83)}=99.53, p<0.001\right)$, main effect of hemisphere $\left(F_{(1)}=\right.$ $31.59, p<0.001)$, and group by region interaction $\left(F_{(5.66)}=2.83\right.$, $p=0.01)$, indicating that there were group differences that varied by region.

To further investigate the group by region interaction, we compared activity to novel stimuli across the groups within each region with follow-up pairwise comparisons (Fig. 3B; Table 4). Overall, there were few significant reductions in activity for novel stimuli in the Tau- OA or Tau + OA groups. Reductions in activity to novel stimuli occurred only in the Tau- OA group (vs YA) in PHC-L and A36-L ( $p$ s <0.05). There were no additional reductions in activity for novel stimuli in the Tau + compared to Tau- OA or YA group ( $p s>0.05$ ). However, certain regions did show hyperactivity to novel stimuli. The Tau- OA group demonstrated hyperactivity to novel stimuli compared to YA in alEC-R $(p<0.05)$. The Tau + OA group demonstrated hyperactivity in hipp-R, pmEC-R, and A35-L compared to Tau- OA, and in hipp-R compared to the YA group ( $p s<0.05)$. In sum, because 
of the lack of reductions in novel activity found in either the Tau- or Tau + OA groups, we concluded that group differences in activity to novel stimuli were not driving our repetition suppression results.

We next assessed group differences for repeated stimuli compared with baseline with repeated measures ANOVAs (Fig. 3C). We found a significant main effect of group $\left(F_{(2)}=5.54\right.$, $p=0.006)$ indicating that, unlike activity to novel stimuli, activity to repeated stimuli differed across groups regardless of MTL region. Generally, activity was greatest in Tau+ OA. We also found a significant main effect of region $\left(F_{(3.28)}=95.20\right.$, $p<0.001)$, main effect of hemisphere $\left(F_{(1)}=40.86, p<0.001\right)$, and group by region interaction $\left(F_{(6.57)}=4.778, p<0.001\right)$, indicating that the level of group differences in activity to repeated stimuli varied across MTL regions.

To further investigate the group by region interaction, we compared activity to repeated stimuli between the groups within each region. Follow-up pairwise comparisons revealed hyperactivity to repeated stimuli for both OA groups in MTL (Fig. 3C; Table 5). Compared to the YA, Tau- OA showed hyperactivity to repeated stimuli in bilateral hippocampus and alEC-R ( $p s<0.05$ ), regions that show deactivation to repeated stimuli within the YA group and tau-related reductions in repetition suppression. Compared to the Tau- OA group, Tau+ OA showed hyperactivity to repeated stimuli in many MTL ROIs, including bilateral hippocampus, bilateral pmEC, A35-L, and bilateral PHC $(p s<0.05)$. Compared to YA, Tau+ OA showed hyperactivity in bilateral hippocampus, alEC-L, and bilateral pmEC $(p s<0.05)$. Overall, the regions demonstrating hyperactivity to repeated stimuli (Fig. $3 C$ ) were consistent with the regions demonstrating reduced repetition suppression (Fig. $3 A$ ). For example, both reduced repetition suppression and hyperactivity to repeated stimuli were found in the Tau-OA group (vs YA) in bilateral hippocampus and alEC-R, and in the Tau+ OA group (vs YA) in the bilateral hippocampus, alEC-L, and bilateral pmEC.

Our finding of hyperactivity to repeated stimuli, with little to no reduction in activity to novel stimuli, suggests the reduced repetition suppression we observed was driven by relative hyperactivity to repeated stimuli compared to that of novel stimuli. To statistically test that hyperactivity to repeated stimuli was driving reduced repetition suppression, we created $z$-scores for activity to novel and repeated stimuli based on the YA sample. Positive $z$-scores could thus be considered "hyperactivity." We then compared $z$-scored activity for novel against repeated stimuli within each OA subject with paired-samples $t$ tests. Within the TauOA group, greater hyperactivity was found for repeated versus novel stimuli in the bilateral hippocampus and alEC-R ( $p$ s $<0.05$; Table 6), the same regions that showed reduced repetition suppression. PHC-L and A36-L also showed greater hyperactivity for repeated than novel stimuli, which may be explained by the fact that these two regions had demonstrated reduced activity to novel stimuli compared to YA. Within the Tau+ OA group, all MTL ROIs showed greater hyperactivity to repeated than to novel stimuli ( $p s<0.05$; Table 6) except A36-L. Thus, our analyses confirmed that hyperactivity was greatest during repeated stimuli, and this change in activity was the most robust driver of reduced repetition suppression.

\section{Hyperactivity is associated with entorhinal tau pathology but not $\mathbf{A} \boldsymbol{\beta}$}

Because cortical tau first deposits in the transentorhinal region (Braak and Braak, 1985, 1991), and hyperactivity was most
Table 6. Hyperactivity to novel and repeated stimuli in the low (Tau-) and high $(\mathrm{Tau}+)$ tau groups

\begin{tabular}{crrrrc}
\hline & First M (SD) & Repeat M (SD) & \multicolumn{1}{c}{$t$} & df & $p$ \\
\hline Tau- OA & & & & & \\
PHC-L & $-0.53(0.65)$ & $-0.43(0.64)$ & 2.24 & 28 & 0.03 \\
PHC-R & $-0.57(1.12)$ & $-0.61(1.14)$ & -0.77 & 28 & 0.45 \\
A36-L & $-0.57(0.83)$ & $-0.35(0.94)$ & 2.76 & 28 & 0.01 \\
A36-R & $-0.42(1.32)$ & $-0.41(1.19)$ & 0.13 & 28 & 0.90 \\
A35-L & $-0.26(0.88)$ & $-0.17(0.86)$ & 1.09 & 28 & 0.22 \\
A35-R & $-0.07(1.04)$ & $-0.06(1.10)$ & 0.06 & 28 & 0.95 \\
pmEC-L & $0.16(0.83)$ & $0.24(0.86)$ & 1.09 & 28 & 0.28 \\
pmEC-R & $-0.36(0.66)$ & $-0.25(0.90)$ & 1.19 & 28 & 0.25 \\
alEC-L & $0.12(1.27)$ & $0.36(1.55)$ & 1.96 & 28 & 0.06 \\
alEC-R & $0.75(1.33)$ & $1.08(1.09)$ & 2.19 & 28 & 0.04 \\
hipp-L & $0.14(0.79)$ & $0.68(0.66)$ & 6.99 & 28 & $<0.001$ \\
hipp-R & $0.12(0.92)$ & $0.59(0.70)$ & 5.40 & 28 & $<0.001$ \\
Tau+ 0A & & & & & \\
PHC-L & $-0.29(0.55)$ & $0.03(0.69)$ & 5.55 & 15 & $<0.001$ \\
PHC-R & $-0.02(1.03)$ & $0.24(1.34)$ & 2.96 & 15 & 0.01 \\
A36-L & $-0.27(1.23)$ & $0.18(1.19)$ & 3.42 & 15 & 0.004 \\
A36-R & $0.01(1.28)$ & $0.17(1.29)$ & 1.48 & 15 & 0.16 \\
A35-L & $0.39(0.86)$ & $0.63(0.90)$ & 2.32 & 15 & 0.04 \\
A35-R & $0.01(0.91)$ & $0.46(0.83)$ & 4.10 & 15 & 0.001 \\
pmEC-L & $0.56(0.74)$ & $0.82(0.88)$ & 2.34 & 15 & 0.03 \\
pmEC-R & $0.38(1.14)$ & $0.83(1.20)$ & 4.60 & 15 & $<0.001$ \\
alEC-L & $0.54(1.07)$ & $0.93(1.18)$ & 3.05 & 15 & 0.008 \\
alEC-R & $0.20(1.67)$ & $0.64(1.25)$ & 2.36 & 15 & 0.03 \\
hipp-L & $0.51(1.28)$ & $1.25(0.92)$ & 4.92 & 15 & $<0.001$ \\
hipp-R & $0.79(1.30)$ & $1.29(0.94)$ & 3.46 & 15 & 0.003 \\
\hline
\end{tabular}

Hyperactivity measures reflect $z$-scores based on the YA group. Hyperactivity differences between novel and repeated stimuli was assessed within each tau group with paired samples $t$ tests. Tau- OA, older adults with low tau; Tau + OA, older adults with high tau; PHC, parahippocampal cortex; A36, area 36 of perirhinal cortex; A35, area 35 of perirhinal cortex; pmEC, posteromedial entorhinal cortex; alEC, anterolateral entorhinal cortex; hipp, hippocampus

strongly found in entorhinal cortex and hippocampus, we next determined whether levels of entorhinal tau were associated with hyperactivity. We extracted mean partial volume corrected FTP SUVR (Baker et al., 2017) from an entorhinal cortex ROI spanning alEC, pmEC, and A35 derived from native space FreeSurfer segmentations. We could not quantify subregional entorhinal tau pathology because of the limited resolution of PET. We focused on hyperactivity in regions that may first be affected by entorhinal tau: A35, alEC, pmEC, and hippocampus. Because of the relative hemispheric similarity of our results, and to reduce the number of statistical tests, we averaged both activity and tau deposition across the two hemispheres. All correlations between activity and entorhinal FTP SUVR controlled for age, sex, and entorhinal cortical thickness to isolate the specific effects of tau over and above basic demographics and neurodegeneration due to other pathologies or vascular disease. Analyses were performed using skipped correlations, which account for bivariate outliers (see Materials and Methods).

Across all OA, entorhinal FTP SUVR was positively correlated with activity for both novel and repeated stimuli in A35 (novel: $r=0.51$; repeated: $r=0.41$; Fig. $6 A$ ), pmEC (novel: $r=0.44$; repeated: $r=0.40$; Fig. $6 B$ ), and hippocampus [novel: $r=0.48$, repeated: $r=0.53$ (Fig. $6 D$ ); all CIs do not include zero; see Table 7]. However, the association between entorhinal FTP SUVR and alEC activity did not reach significance [novel: $r=0.19$; repeated: $r=0.17$ (Fig. 6C); CIs cross zero; see Table 7].

Next, we tested whether $\mathrm{A} \beta$, quantified with a global measure of cortical PiB DVR (Mormino et al., 2012b; Villeneuve et al., 2015), was also associated with activation. There was no association between global PiB DVR and activity levels in any of the 
A

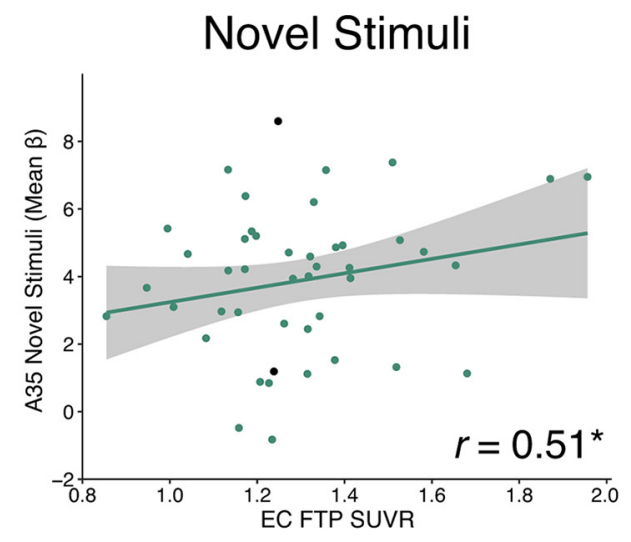

B

A35

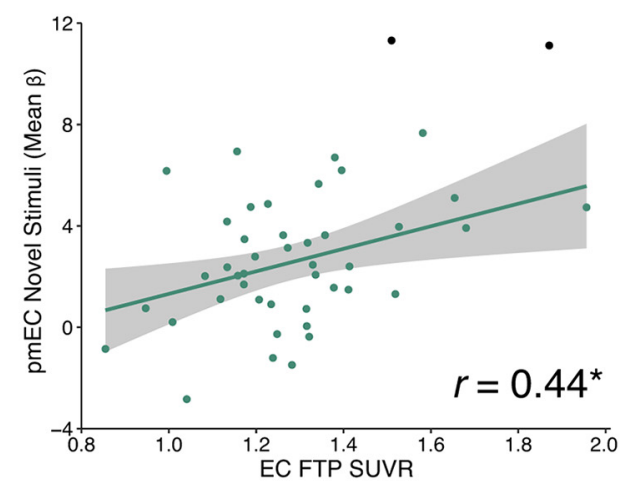

C

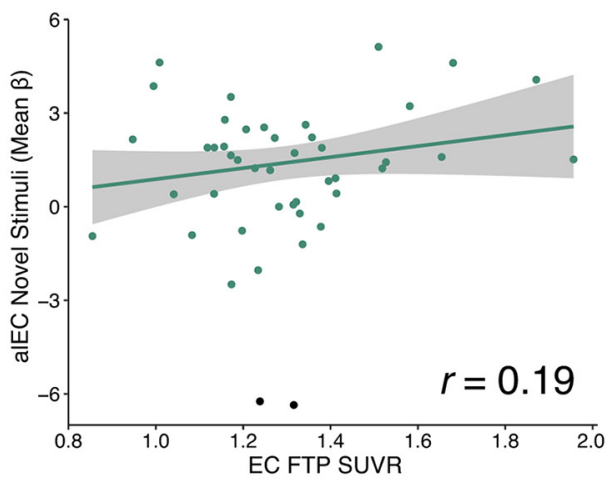

D

alEC

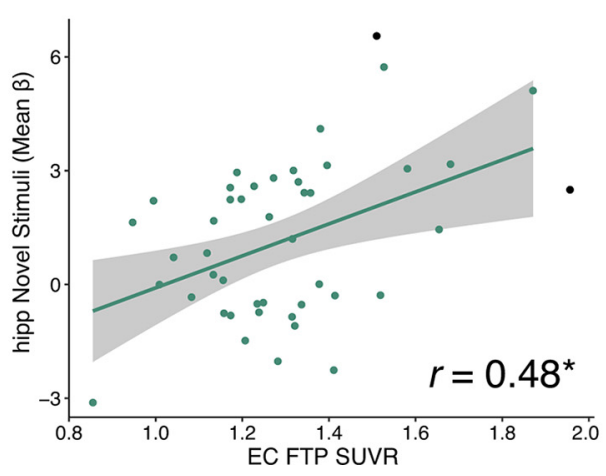

Repeated Stimuli
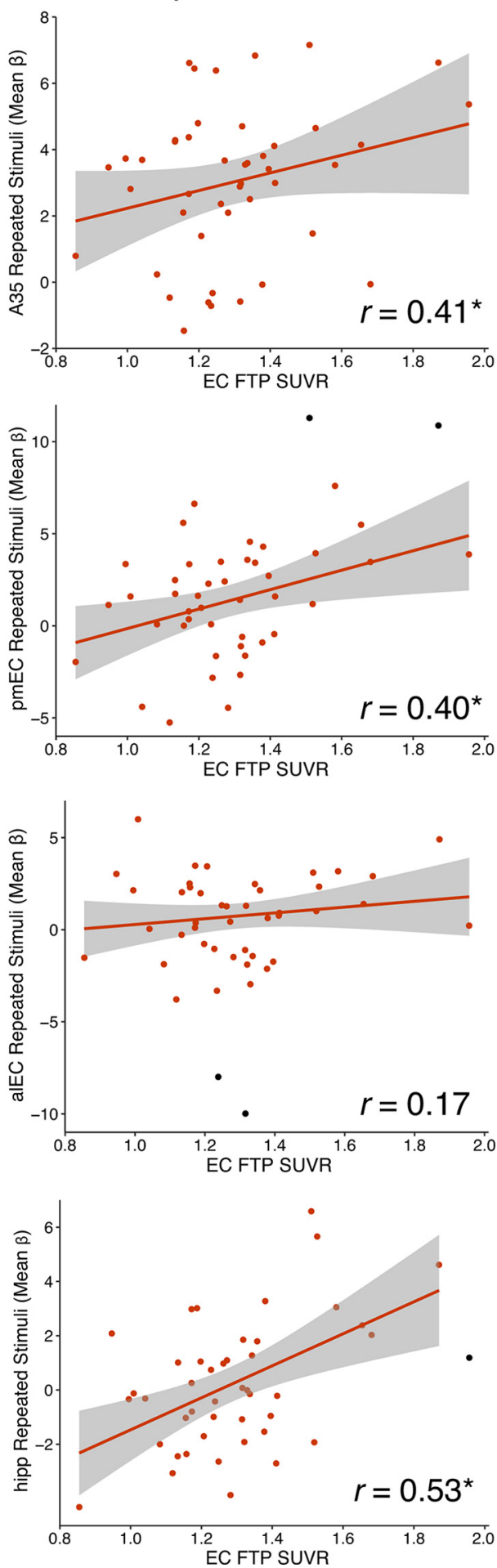

Figure 6. Increased activation to novel and repeated stimuli is associated with entorhinal tau pathology in OAs. Mean FTP SUVR of native space entorhinal cortex (EC) was extracted and partial volume corrected for each $\mathrm{OA}$ subject. To obtain measures of activity, the mean $\beta$ value for each ROI was extracted for novel and repeated stimuli (compared to perceptual baseline). Robust regressions were performed to reduce the effects of outliers. All correlations controlled for age, sex, and EC thickness (raw data shown). EC FTP SUVR was associated with increased activity to both novel and repeated stimuli in A35 $(\boldsymbol{A})$, pmEC $(\boldsymbol{B})$, and hippocampus $(\boldsymbol{D})$, but not alEC $(\boldsymbol{C}) ;{ }^{*}$ indicates significant skipped correlations (Cl does not include zero). Black data points were identified as bivariate outliers and removed in the skipped correlation.

ROIs for novel or repeated stimuli (CIs crossed zero; see Table 7 ). We also found no significant interactions between entorhinal FTP SUVR and $\mathrm{A} \beta$ status using linear regression models predicting to activity in each ROI, including age and sex as covariates (interaction $p s>0.05$; see Table 7 ).

\section{Associations with cognitive performance}

Finally, we investigated whether our measures of MTL repetition suppression activity and entorhinal tau deposition in OA were related to task performance (corrected hit rate) or an episodic memory composite from neuropsychological tests. We first 
Table 7. Associations between activation to novel and repeated stimuli with measures of entorhinal tau and global $A \beta$

\begin{tabular}{|c|c|c|c|c|c|c|c|}
\hline \multirow[b]{2}{*}{ ROI } & \multicolumn{2}{|c|}{ EC FTP SUVR $^{a}$} & \multicolumn{2}{|c|}{ Global PiB DVR } & \multicolumn{3}{|c|}{ EC FTP $\times A \beta$ Status $^{c}$} \\
\hline & $r$ & $\mathrm{Cl}$ & $r$ & $\mathrm{Cl}$ & $\beta$ coefficient & $t$ & $p$ \\
\hline \multicolumn{8}{|c|}{ Novel stimuli } \\
\hline A35 & $0.51^{*}$ & {$[0.29,0.70]$} & 0.05 & {$[-0.31,0.39]$} & 0.99 & 0.34 & 0.73 \\
\hline pmEC & $0.44^{*}$ & {$[0.18,0.65]$} & 0.21 & {$[-0.08,0.47]$} & -0.17 & -0.04 & 0.97 \\
\hline alEC & 0.19 & {$[-0.04,0.43]$} & 0.04 & {$[-0.24,0.32]$} & -2.38 & -0.67 & 0.51 \\
\hline hipp & $0.48^{*}$ & {$[0.19,0.68]$} & 0.17 & {$[-0.14,0.43]$} & -4.00 & -1.37 & 0.18 \\
\hline \multicolumn{8}{|c|}{ Repeated stimuli } \\
\hline A35 & $0.41^{*}$ & {$[0.19,0.61]$} & 0.19 & {$[-0.11,0.47]$} & 0.49 & 0.16 & 0.88 \\
\hline $\mathrm{pmEC}$ & $0.40^{*}$ & {$[0.13,0.60]$} & 0.20 & {$[-0.11,0.46]$} & 0.001 & 0.00 & 0.99 \\
\hline alEC & 0.17 & {$[-0.09,0.44]$} & 0.02 & {$[-0.27,0.33]$} & -0.86 & -0.19 & 0.85 \\
\hline hipp & $0.53^{*}$ & {$[0.27,0.73]$} & 0.17 & {$[-0.16,0.48]$} & -3.73 & -1.14 & 0.26 \\
\hline
\end{tabular}

$a, b$ Skipped Pearson's $r$ and Cls were calculated with robust regression.

$a$ Controlling for effects of age, sex, and EC cortical thickness. $b$ Controlling for effects of age and sex.

$c$ Interactions were calculated using linear models, which predicted activity in each ROI. The interaction term of EC FTP $\times$ A $\beta$ status (values shown), age, and sex were included as predictors in the model. ${ }^{*} \mathrm{Cl}$ does not cross zero, indicating significance of the skipped correlation. A35, area 35 of perirhinal cortex; pmEC, posteromedial entorhinal cortex; alEC, anterolateral entorhinal cortex; hipp, hippocampus.

assessed the relationship between entorhinal tau pathology and cognitive performance, using skipped correlations and controlling for effects of age, sex, and education. There was a significant negative correlation between entorhinal FTP and the episodic memory composite (skipped Pearson's $r=-0.33$, CI $[-0.55$, $-0.05]$ ), but no significant association between entorhinal FTP and corrected hit rate on the task $(r=-0.10,[-0.31,0.14])$. We next performed skipped correlations between repetition suppression activity in each MTL region with cognitive performance, including the same covariates. However, there was no significant relationship between repetition suppression activity and either corrected hit rate ( $r$ s ranged $-0.02-0.12$; all CIs crossed zero) or the episodic memory composite ( $r$ ranged $-0.06-0.13$; all CIs crossed zero) in any region.

\section{Discussion}

We demonstrate that reduced repetition suppression activity in the MTL is related to tau pathology in cognitively normal OAs. Although our data are cross-sectional, our results suggest that reductions are limited to the alEC and hippocampus in the context of low tau pathology restricted to these regions, but may expand to encompass the rest of the MTL when tau pathology reaches a more advanced stage. Reduced repetition suppression was driven by hyperactivity to repeated stimuli, rather than hypoactivity to novel stimuli. Tau pathology in entorhinal cortex, but not global $\mathrm{A} \beta$, was related to increased activation to both novel and repeated stimuli in entorhinal regions and hippocampus. We conclude that tau deposition in cognitively normal OAs is related to hyperactivity, leading to failures of repetition suppression activity within MTL.

We classified OA participants as low tau (Tau-), with either no measurable tau or tau deposition restricted to entorhinal/hippocampus, or high tau $(\mathrm{Tau}+)$, with more advanced tau pathology expanded to other temporal and limbic regions. Reduced repetition suppression activity in the Tau- OA group, compared to a young control group, occurred focally in alEC and hippocampus. This finding supported our hypothesis that activity reductions in low tau individuals, where tau deposition is nearly universal in the transentorhinal/anterolateral entorhinal region, would specifically co-localize with the earliest cortical tau pathology (Braak and Braak, 1985, 1991). Reduced activity within alEC and hippocampus suggests that tau deposition may underlie activity changes in normal aging. This is supported by prior studies that have demonstrated that the alEC is particularly vulnerable to the effects of age and preclinical AD (Khan et al., 2014; Olsen et al., 2017; Reagh et al., 2018). Hippocampal activity (Marks et al., 2017; Berron et al., 2019; Huijbers et al., 2019) and regional homogeneity of fMRI resting state signal (Harrison et al., 2019) have also been found to be associated with tau pathology in aging, consistent with our findings.

We next hypothesized reduced repetition suppression across the MTL in the Tau+ OA group, as this group is at a more advanced stage of pathology possibly reflecting preclinical $\mathrm{AD}$ (Jagust, 2018). Confirming our hypothesis, the majority of MTL regions demonstrated reduced repetition suppression in the Tau + OA group. While alEC activity was not further reduced from the Tau- to Tau + OA group, hippocampal activity continued to decrease with increasing tau pathology. This continual reduction in hippocampal repetition suppression is consistent with theories of hippocampal disconnection as a key mechanism of disease progression (Hyman et al., 1984).

To probe this reduction in repetition suppression activity, we determined that hyperactivity to repeated stimuli, rather than hypoactivity to novel stimuli, was driving reduced repetition suppression activity. While entorhinal tau was correlated with increased activation to both novel and repeated stimuli, a direct comparison showed greater hyperactivity for repeated stimuli compared to novel stimuli. As activity should reduce with repeated presentations of stimuli for successful repetition suppression, this result is consistent with findings of $\mathrm{A} \beta$-related deactivation failures in the default mode network during memory encoding in cognitively normal OAs (Sperling et al., 2009; Huijbers et al., 2014) and patients with AD (Celone et al., 2006; Petrella et al., 2007; Pihlajamaki et al., 2008). Together, our findings suggest that task components where neuronal deactivation is optimal to processing may be more vulnerable to hyperactivity, as there may be a ceiling to activity that limits the extent to which an already active system can hyperactivate. Therefore, tau deposition functions to generally increase the system gain that disproportionately affects processing of repeated stimuli, possibly decreasing neural discrimination between novel and repeated stimuli.

Entorhinal tau, which occurs in almost all OAs (Braak and Braak, 1997), was associated with increased activation in A35, pmEC, and hippocampus. A recent study did not observe associations between entorhinal tau and hippocampal hyperactivity, concluding that the spread of tau outside of the MTL is necessary for tau-related activity changes (Huijbers et al., 2019). Our finding reveals that entorhinal tau is not benign, and is related to activity changes in key MTL subregions. The lack of association between entorhinal tau and alEC hyperactivity was unexpected due to the group differences in this region. Because alEC is one of the first cortical regions to deposit tau (Braak and Braak, 1985, 1991), its hyperactivity may have reached a ceiling, and thus did not show a continuous relationship with current levels of entorhinal tau. Alternatively, tau-related hyperactivity in alEC may yield to hypoactivity as neurodegenerative processes begin to occur (Dickerson et al., 2005; O'Brien et al., 2010).

Reduced repetition suppression activity in the MTL is ubiquitous, previously found in patients with $\mathrm{AD}$ (Sperling et al., 2003; Golby et al., 2005; Pihlajamäki et al., 2011), MCI (Johnson et al., 2004), and across cognitively normal and impaired OAs (Düzel et al., 2018). Our results expand on these observations by linking this phenomenon to tau deposition. Our findings are also consistent with recent studies demonstrating associations between 
tau and hippocampal hyperactivity in aging (Marks et al., 2017; Berron et al., 2019; Huijbers et al., 2019), although we expand on this literature by finding that other vulnerable MTL regions show tau-related activity changes in aging. This study also extends our previous observation of increased activity during object and scene processing in this cohort (Maass et al., 2019) by examining trial-level activity in MTL subregions and linking the increased activity to reduced repetition suppression. Our findings of MTL hyperactivity are also consistent with studies demonstrating hyperconnectivity between MTL subregions in MCI (Das et al., 2013; Pasquini et al., 2016; Berron et al., 2020). Finally, consistent with other recent studies (Berron et al., 2019; Huijbers et al., 2019; Maass et al., 2019), we did not observe a relationship between global A $\beta$ and MTL activity, supporting the stronger role of tau on local activity in the MTL.

In contrast to our findings of tau-related hyperactivity, findings from animal models have suggested that tau is associated with hypoactivity and silencing of neurons (Angulo et al., 2017; Busche et al., 2019). While disconnection from entorhinal cortex and a lack of inhibition can explain tau-related hippocampal hyperactivity (Hyman et al., 1984), tau-related hyperactivity in other cortical regions is harder to reconcile with this animal literature. An alternative explanation of our findings is rather than tau inducing hyperactivity, hyperactivity may lead to increased tau deposition. Animal models demonstrate that increased neuronal activity leads to the release and spread of pathological tau (Pooler et al., 2013; Wu et al., 2016). It is possible that OAs with greater levels of MTL activity, perhaps because of compensatory mechanisms, produce more pathological tau. Functional connectivity driving tau spread has also been supported by recent findings in human neuroimaging studies (Adams et al., 2019; Franzmeier et al., 2019; Vogel et al., 2020). Longitudinal studies will help elucidate the temporal relationship between tau deposition and hyperactivity.

Finally, we did not observe any associations between repetition suppression activity and cognitive performance. It is possible that repetition suppression activity may not express itself behaviorally as explicit changes to task performance or episodic memory. Because our sample is high-performing and well-educated, there may have not been enough variation in scores to observe an association, or brain resilience may overcome the effects of tau pathology. Relationships between activity, tau, and cognitive performance should be further investigated to better understand activity changes in the context of behavior.

There are several limitations to this study. Because some MTL regions preferentially process objects versus scenes (Ranganath and Ritchey, 2012; Berron et al., 2018), we also analyzed each stimulus type separately, producing similar results. Additionally, we were not able to measure tau deposition within specific MTL subregions (e.g., alEC or A35) because of the resolution of PET, or hippocampal tau because of effects of off-target FTP binding in the adjacent choroid plexus (Baker et al., 2017). Because of the resolution and use of smoothing on the functional data, in addition to the motion threshold we selected, some signal blurring between neighboring MTL subregions may have occurred. There may also be some uncertainty of the precise border between alEC and pmEC. Finally, while we carefully removed areas of low signal before analyses, the alEC is particularly vulnerable to signal drop out, and alEC-tau null results may be partly because of loss of signal.

In conclusion, we demonstrate that pathological tau accumulation is associated with hyperactivity in MTL, which specifically affects the ability to deactivate to repeated stimuli and reduces repetition suppression. Reductions in repetition suppression activity were found focally in alEC and hippocampus in OAs with low tau levels, and across the MTL in the context of high tau pathology. Entorhinal tau pathology, found in nearly all OAs, was related to a general increase in activation across novel and repeated stimuli, while $\mathrm{A} \beta$ did not demonstrate any associations with MTL activation. The directionality of associations between tau and hyperactivity is still an open question that needs to be further explored in longitudinal studies, as determining the causality would help develop interventions to prevent tau progression and cognitive decline.

\section{References}

Adams JN, Maass A, Harrison TM, Baker SL, Jagust WJ (2019) Cortical tau deposition follows patterns of entorhinal functional connectivity in aging. Elife 8:e49132.

Angulo SL, Orman R, Neymotin SA, Liu L, Buitrago L, Cepeda-Prado E, Stefanov D, Lytton WW, Stewart M, Small SA, Duff KE, Moreno H (2017) Tau and amyloid-related pathologies in the entorhinal cortex have divergent effects in the hippocampal circuit. Neurobiol Dis 108:261-276.

Avants BB, Yushkevich P, Pluta J, Minkoff D, Korczykowski M, Detre J, Gee JC (2010) The optimal template effect in hippocampus studies of diseased populations. Neuroimage 49:2457-2466.

Baker SL, Maass A, Jagust WJ (2017) Considerations and code for partial volume correcting [18F]-AV-1451 tau PET data. Data Brief 15:648-657.

Bakker A, Krauss GL, Albert MS, Speck CL, Jones LR, Stark CE, Yassa MA, Bassett SS, Shelton AL, Gallagher M (2012) Reduction of hippocampal hyperactivity improves cognition in amnestic mild cognitive impairment. Neuron 74:467-474.

Berron D, Vieweg P, Hochkeppler A, Pluta JB, Ding SL, Maass A, Luther A, Xie L, Das SR, Wolk DA, Wolbers T, Yushkevich PA, Düzel E, Wisse LEM (2017) A protocol for manual segmentation of medial temporal lobe subregions in 7 Tesla MRI. NeuroImage Clin 15:466-482.

Berron D, Neumann K, Maass A, Schütze H, Fliessbach K, Kiven V, Jessen F, Sauvage M, Kumaran D, Düzel E (2018) Age-related functional changes in domain-specific medial temporal lobe pathways. Neurobiol Aging 65:86-97.

Berron D, Cardenas-Blanco A, Bittner D, Metzger CD, Spottke A, Heneka MT, Fließbach K, Schneider A, Teipel SJ, Wagner M, Speck O, Jessen F, Düzel E (2019) Higher CSF tau levels are related to hippocampal hyperactivity and object mnemonic discrimination in older adults. J Neurosci 39:8788-8797.

Berron D, van Westen D, Ossenkoppele R, Strandberg O, Hansson O (2020) Medial temporal lobe connectivity and its associations with cognition in early Alzheimer's disease. Brain 143:1233-1248.

Braak H, Braak E (1985) On areas of transition between entorhinal allocortex and temporal isocortex in the human brain. Normal morphology and lamina-specific pathology in Alzheimer's disease. Acta Neuropathol 68:325-332.

Braak H, Braak E (1991) Neuropathological stageing of Alzheimer-related changes. Acta Neuropathol 82:239-259.

Braak H, Braak E (1997) Frequency of stages of Alzheimer-related lesions in different age categories. Neurobiol Aging 18:351-357.

Busche MA, Wegmann S, Dujardin S, Commins C, Schiantarelli J, Klickstein N, Kamath TV, Carlson GA, Nelken I, Hyman BT (2019) Tau impairs neural circuits, dominating amyloid- $\beta$ effects, in Alzheimer models in vivo. Nat Neurosci 22:57-64.

Celone KA, Calhoun VD, Dickerson BC, Atri A, Chua EF, Miller SL, DePeau K, Rentz DM, Selkoe DJ, Blacker D, Albert MS, Sperling RA (2006) Alterations in memory networks in mild cognitive impairment and Alzheimer's disease: an independent component analysis. J Neurosci 26:10222-10231.

Das SR, Pluta J, Mancuso L, Kliot D, Orozco S, Dickerson BC, Yushkevich PA, Wolk DA (2013) Increased functional connectivity within medial temporal lobe in mild cognitive impairment. Hippocampus 23:1-6.

Dickerson BC, Salat DH, Greve DN, Chua EF, Rand-Giovannetti E, Rentz DM, Bertram L, Mullin K, Tanzi RE, Blacker D, Albert MS, Sperling RA (2005) Increased hippocampal activation in mild cognitive impairment compared to normal aging and AD. Neurology 65:404-411. 
Düzel E, Berron D, Schütze H, Cardenas-Blanco A, Metzger C, Betts M, Ziegler G, Chen Y, Dobisch L, Bittner D, Glanz W, Reuter M, Spottke A, Rudolph J, Brosseron F, Buerger K, Janowitz D, Fliessbach K, Heneka M, Laske C, et al. (2018) CSF total tau levels are associated with hippocampal novelty irrespective of hippocampal volume. Alzheimers Dement (Amst) 10:782-790.

Franzmeier N, Rubinski A, Neitzel J, Kim Y, Damm A, Na DL, Kim HJ, Lyoo $\mathrm{CH}$, Cho H, Finsterwalder S, Duering M, Seo SW, Ewers M; Alzheimer's Disease Neuroimaging Initiative (2019) Functional connectivity associated with tau levels in ageing. Brain 142:1093-1015.

Golby A, Silverberg G, Race E, Gabrieli S, O’Shea J, Knierim K, Stebbins G, Gabrieli J (2005) Memory encoding in Alzheimer's disease: an fMRI study of explicit and implicit memory. Brain 128:773-787.

Greenhouse SW, Geisser S (1959) On methods in the analysis of profile data. Psychometrika 24:95-112.

Grill-Spector K, Henson R, Martin A (2006) Repetition and the brain: neural models of stimulus-specific effects. Trends Cogn Sci 10:14-23.

Harrison TM, Maass A, Adams JN, Du R, Baker SL, Jagust WJ (2019) Tau deposition is associated with functional isolation of the hippocampus in aging. Nat Commun 10:4900.

Huijbers W, Mormino EC, Wigman SE, Ward AM, Vannini P, McLaren DG, Becker JA, Schultz AP, Hedden T, Johnson KA, Sperling RA (2014) Amyloid deposition is linked to aberrant entorhinal activity among cognitively normal older adults. J Neurosci 34:5200-5210.

Huijbers W, Mormino EC, Schultz AP, Wigman S, Ward AM, Larvie M, Amariglio RE, Marshall GA, Rentz DM, Johnson KA, Sperling RA (2015) Amyloid- $\beta$ deposition in mild cognitive impairment is associated with increased hippocampal activity, atrophy and clinical progression. Brain 138:1023-1035.

Huijbers XW, Schultz AP, Papp KV, Lapoint MR, Hanseeuw X, Chhatwal XJP, Hedden T, Johnson XA, Sperling XRA (2019) Tau accumulation in clinically normal older adults is associated with hippocampal hyperactivity. J Neurosci 39:548-556.

Hyman BT, Van Hoesen GW, Damasio AR, Barnes CL (1984) Alzheimer's disease: cell-specific pathology isolates the hippocampal formation. Science 225:1168-1170.

Jagust W (2018) Imaging the evolution and pathophysiology of Alzheimer disease. Nat Rev Neurosci 19:687-700.

Johnson SC, Baxter LC, Susskind-Wilder L, Connor DJ, Sabbagh MN, Caselli RJ (2004) Hippocampal adaptation to face repetition in healthy elderly and mild cognitive impairment. Neuropsychologia 42:980-989.

Khan UA, Liu L, Provenzano FA, Berman DE, Profaci CP, Sloan R, Mayeux R, Duff KE, Small SA (2014) Molecular drivers and cortical spread of lateral entorhinal cortex dysfunction in preclinical Alzheimer's disease. Nat Neurosci 17:304-311.

Lemieux L, Salek-Haddadi A, Lund TE, Laufs H, Carmichael D (2007) Modelling large motion events in fMRI studies of patients with epilepsy. Magn Reson Imaging 25:894-901.

Libby LA, Ekstrom AD, Ragland JD, Ranganath C (2012) Differential connectivity of perirhinal and parahippocampal cortices within human hippocampal subregions revealed by high-resolution functional imaging. J Neurosci 32:6550-6560

Logan J (2000) Graphical analysis of PET data applied to reversible and irreversible tracers. Nucl Med Biol 27:661-670.

Maass A, Berron D, Libby L, Ranganath C, Düzel E (2015) Functional subregions of the human entorhinal cortex. Elife 4:e06426.

Maass A, Landau S, Baker SL, Horng A, Lockhart SN, La Joie R, Rabinovici GD, Jagust WJ; Alzheimer's Disease Neuroimaging Initiative (2017) Comparison of multiple tau-PET measures as biomarkers in aging and Alzheimer's disease. Neuroimage 157:448-463.

Maass A, Lockhart SN, Harrison TM, Bell RK, Mellinger T, Swinnerton K, Baker SL, Rabinovici GD, Jagust WJ (2018) Entorhinal tau pathology, episodic memory decline and neurodegeneration in aging. J Neurosci 38:530-543.

Maass A, Berron D, Harrison TM, Adams JN, La Joie R, Baker SL, Mellinger TJ, Bell RK, Swinnerton KN, Inglis B, Rabinovici GD, Düzel E, Jagust WJ (2019) Alzheimer's pathology targets distinct memory networks in the ageing. brain 142:2492-2509.

Marks SM, Lockhart SN, Baker SL, Jagust WJ (2017) Tau and $\beta$-amyloid are associated with medial temporal lobe structure, function and memory encoding in normal aging. J Neurosci 37:3769-3716.
Mathis CA, Wang Y, Holt DP, Huang GF, Debnath ML, Klunk WE (2003) Synthesis and evaluation of 11C-labeled 6-substituted 2-arylbenzothiazoles as amyloid imaging agents. J Med Chem 46:2740-2754.

Mormino EC, Brandel MG, Madison CM, Marks S, Baker SL, Jagust WJ (2012a) A $\beta$ deposition in aging is associated with increases in brain activation during successful memory encoding. Cereb Cortex 22:1813-1823.

Mormino EC, Brandel MG, Madison CM, Rabinovici GD, Marks S, Baker SL, Jagust WJ (2012b) Not quite PIB-positive, not quite PIB-negative: slight PIB elevations in elderly normal control subjects are biologically relevant. Neuroimage 59:1152-1160.

O'Brien JL, O'Keefe KM, LaViolette PS, DeLuca AN, Blacker D, Dickerson BC, Sperling RA (2010) Longitudinal fMRI in elderly reveals loss of hippocampal activation with clinical decline. Neurology 74:19691976.

Olsen RK, Yeung LK, Noly-Gandon A, D’Angelo MC, Kacollja A, Smith VM, Ryan JD, Barense MD (2017) Human anterolateral entorhinal cortex volumes are associated with cognitive decline in aging prior to clinical diagnosis. Neurobiol Aging 57:195-205.

Pasquini L, Scherr M, Tahmasian M, Myers NE, Ortner M, Kurz A, Förstl H, Zimmer C, Grimmer T, Akhrif A, Wohlschläger AM, Riedl V, Sorg C (2016) Increased intrinsic activity of medial-temporal lobe subregions is associated with decreased cortical thickness of medial-parietal areas in patients with Alzheimer's disease dementia. J Alzheimers Dis 51:313326.

Pernet CR, Wilcox R, Rousselet GA (2013) Robust correlation analyses: false positive and power validation using a new open source MATLAB toolbox. Front Psychol 3:1-18.

Petrella JR, Prince SE, Wang L, Hellegers C, Doraiswamy PM (2007) Prognostic value of posteromedial cortex deactivation in mild cognitive impairment. PLoS One 2:e1104.

Pihlajamäki M, Depeau KM, Blacker D, Sperling RA (2008) Impaired medial temporal repetition suppression is related to failure of parietal deactivation in Alzheimer disease. Am J Geriatr Psychiatry 16:283-292.

Pihlajamäki M, O’Keefe K, O’Brien J, Blacker D, Sperling RA (2011) Failure of repetition suppression and memory encoding in aging and Alzheimer's disease. Brain Imaging Behav 5:36-44.

Pooler AM, Phillips EC, Lau DHW, Noble W, Hanger DP (2013) Physiological release of endogenous tau is stimulated by neuronal activity. EMBO Rep 14:389-394.

Power JD, Schlaggar BL, Petersen SE (2015) Recent progress and outstanding issues in motion correction in resting state fMRI. Neuroimage 105:536551

Price JC, Klunk WE, Lopresti BJ, Lu X, Hoge JA, Ziolko SK, Holt DP, Meltzer CC, DeKosky ST, Mathis CA (2005) Kinetic modeling of amyloid binding in humans using PET imaging and Pittsburgh Compound-B. J Cereb Blood Flow Metab 25:1528-1547.

Ranganath C, Ritchey M (2012) Two cortical systems for memory-guided behaviour. Nat Rev Neurosci 13:713-726.

Reagh ZM, Noche JA, Tustison NJ, Delisle D, Murray EA, Yassa MA (2018) Functional imbalance of anterolateral entorhinal cortex and hippocampal dentate/CA3 underlies age-related object pattern separation deficits. Neuron 97:1187-1198.e4.

Rousset OG, Ma Y, Evans AC (1998) Correction for partial volume effects in PET: principle and validation. J Nucl Med 39:904-911.

Schöll M, Lockhart SN, Schonhaut DR, O’Neil JP, Janabi M, Ossenkoppele R, Baker SL, Vogel JW, Faria J, Schwimmer HD, Rabinovici GD, Jagust WJ (2016) PET imaging of tau deposition in the aging human brain. Neuron 89:971-982.

Sperling RA, Bates JF, Chua EF, Cocchiarella AJ, Rentz DM, Rosen BR, Schacter DL, Albert MS (2003) fMRI studies of associative encoding in young and elderly controls and mild Alzheimer's disease. J Neurol Neurosurg Psychiatry 74:44-50.

Sperling RA, Laviolette PS, O’Keefe K, O’Brien J, Rentz DM, Pihlajamaki M, Marshall G, Hyman BT, Selkoe DJ, Hedden T, Buckner RL, Becker JA, Johnson KA (2009) Amyloid deposition is associated with impaired default network function in older persons without dementia. Neuron 63:178-188

Villeneuve S, Rabinovici GD, Cohn-Sheehy BI, Madison C, Ayakta N, Ghosh PM, La Joie R, Arthur-Bentil SK, Vogel JW, Marks SM, Lehmann M, Rosen HJ, Reed B, Olichney J, Boxer AL, Miller BL, Borys E, Jin LW, Huang EJ, Grinberg LT, et al. (2015) Existing Pittsburgh compound-B 
positron emission tomography thresholds are too high: statistical and pathological evaluation. Brain 138:2020-2033.

Vogel JW, Iturria-Medina Y, Strandberg OT, Smith R, Levitis E, Evans AC, Hansson O; Alzheimer's Disease Neuroimaging Initiative (2020) Spread of pathological tau proteins through communicating neurons in human Alzheimer's disease. Nat Commun 11:2612.

Wilcox RR (2004) Inferences based on a skipped correlation coefficient. J Appl Stat 31:131-143.

Wu JW, Hussaini SA, Bastille IM, Rodriguez GA, Mrejeru A, Rilett K, Sanders DW, Cook C, Fu H, Boonen RACM, Herman M, Nahmani E, Emrani S, Figueroa YH, Diamond MI, Clelland CL, Wray S, Duff KE (2016) Neuronal activity enhances tau propagation and tau pathology in vivo. Nat Neurosci 19:1085-1092.
Yassa MA, Stark SM, Bakker A, Albert MS, Gallagher M, Stark CEL (2010) High-resolution structural and functional MRI of hippocampal CA3 and dentate gyrus in patients with amnestic mild cognitive impairment. Neuroimage 51:1242-1252.

Yassa MA, Lacy JW, Stark SM, Albert MS, Gallagher M, Stark CEL (2011) Pattern separation deficits associated with increased hippocampal CA3 and dentate gyrus activity in nondemented older adults. Hippocampus 21:968-979.

Yushkevich PA, Pluta JB, Wang H, Xie L, Ding SL, Gertje EC, Mancuso L, Kliot D, Das SR, Wolk DA (2015) Automated volumetry and regional thickness analysis of hippocampal subfields and medial temporal cortical structures in mild cognitive impairment. Hum Brain Mapp 36:258-287. 